\title{
Heating and cooling processes in disks ${ }^{\star}$
}

\author{
Peter Woitke \\ ${ }^{1}$ SUPA, School of Physics \& Astronomy, University of St. Andrews, North Haugh, St. Andrews KY16 9SS, UK
}

\begin{abstract}
This chapter summarises current theoretical concepts and methods to determine the gas temperature structure in protoplanetary disks by balancing all relevant heating and cooling rates. The processes considered are non-LTE line heating/cooling based on the escape probability method, photo-ionisation heating and recombination cooling, free-free heating/cooling, dust thermal accommodation and high-energy heating processes such as X-ray and cosmic ray heating, dust photoelectric and PAH heating, a number of particular follow-up heating processes starting with the UV excitation of $\mathrm{H}_{2}$, and the release of binding energy in exothermal reactions. The resulting thermal structure of protoplanetary disks is described and discussed.
\end{abstract}

\section{Introduction}

The temperature of the gas in protoplanetary disks is important for its chemical composition, the production of emission lines connected to the observability of the gas phase, and the gas temperature is essential to predict the pressure structure in disks which determines the vertical stratification and shape of the disk. Therefore, the study of heating and cooling processes is an integral part of disk modelling, one of the four fundamental ingredients for astrophysical modelling, besides radiative transfer, chemistry, and (magneto-)hydrodynamics.

\subsection{Internal energy, thermal stability, and cooling timescale}

In order to determine the temperature of the gas, we consider the first law of thermodynamics

$$
\frac{d(\rho e)}{d t}=-p \frac{d V}{d t}+\sum_{i} \rho \Gamma_{i}-\sum_{k} \rho \Lambda_{k},
$$

where $e\left[\mathrm{erg} / \mathrm{cm}^{3}\right]$ is the internal energy, $p\left[\mathrm{erg} / \mathrm{cm}^{3}\right]$ the gas pressure, $V=1 / \rho\left[\mathrm{cm}^{3} / \mathrm{g}\right]$ the specific volume, $\rho\left[\mathrm{g} / \mathrm{cm}^{3}\right]$ the mass density, and $\Gamma_{i}$ ("gain") and $\Lambda_{k}$ ("loss") are the various heating and cooling rates per volume $\left[\mathrm{erg} / \mathrm{cm}^{3} / \mathrm{s}\right]$.

The $-p d V$ work is usually not important in disks, because there is no expansion or contraction of the gas in stable Keplerian orbits. However, conditions are different for e.g. the accretion columns (usually not covered by disk models), or in case of spiral waves in gravitationally unstable disks (Ilee

$\star 10^{\text {th }}$ Lecture from Summer School "Protoplanetary Disks: Theory and Modelling Meet Observations" 
et al. 2011). Spiral waves cause moderate shock waves which again and again compress and mix the gas. In-between the shock compressions, the gas will re-expand.

In order to assess the importance of the $-p d V$ work we can compare the cooling relaxation timescale $\tau_{\text {cool }}$ with the dynamical timescale $\tau_{\text {dyn }}$, given e.g. by the evolutionary timescale in case of a stable 2D disk in Keplerian rotation, or by the orbital timescale in case of a strongly time-dependent situation like a disk with spiral waves. In most cases, heating/cooling will be rapid and we will find $\tau_{\text {cool }} \ll \tau_{\text {dyn }}$, so the $-p d V$ work can be neglected, and Eq. (1) simplifies to

$$
\frac{d e}{d t}=Q=\sum_{i} \Gamma_{i}-\sum_{k} \Lambda_{k}=0,
$$

which states the condition of thermal energy equilibrium. Studying small deviations from that equilibrium, $e=e_{0}+\delta e$ with $Q\left(e_{0}\right)=0$ and small $\delta e$, we find $d e / d t=d(\delta e) / d t=Q(e) \approx Q\left(e_{0}\right)+\left.\frac{\partial Q}{\partial e}\right|_{e_{0}} \delta e$, thus $d \ln (\delta e) / d t=\left.\frac{\partial Q}{\partial e}\right|_{e_{0}}=$ const, and the cooling timescale can be identified as $\tau_{\text {cool }}=-1 /\left.\frac{\partial Q}{\partial e}\right|_{e_{0}}$, or

$$
\tau_{\text {cool }}=-\left.\frac{\partial e}{\partial T_{\mathrm{g}}}\right|_{T_{\mathrm{g}, 0}}\left|\frac{\partial Q}{\partial T_{\mathrm{g}}}\right|_{T_{\mathrm{g}, 0}},
$$

where $T_{\mathrm{g}}$ is the gas temperature and $T_{\mathrm{g}, 0}$ the gas temperature in thermal balance. While deriving Eq. (3) we have selected $T_{\mathrm{g}}$ and $V$ as state variables to express $e=e\left(T_{\mathrm{g}}, V\right)$ and $Q=Q\left(T_{\mathrm{g}}, V\right)$, and considered an isochoric process $\delta V=0$, so there is no $-p d V$ work. Note that Eq. (2), in principle, may have several temperature solutions $T_{\mathrm{g}, 0}$ ("thermal bifurcations"). Since $\left.\frac{\partial e}{\partial T_{\mathrm{g}}}\right|_{T_{\mathrm{g}, 0}}$ is always positive, thermal stability requires $\left.\frac{\partial Q}{\partial T_{\mathrm{g}}}\right|_{T_{\mathrm{g}, 0}}<0$.

\subsection{The pools of energy}

Before we start evaluating the internal energy $e$ and the net heating/cooling rate $Q$, we first have to agree on the thermodynamical concept and the definition of the internal energy (see, e.g. Rybicki \& Lightman 1979; Shu 1992). In astrophysics, there are two competing approaches, see Fig. 1. The first

\section{LTE}

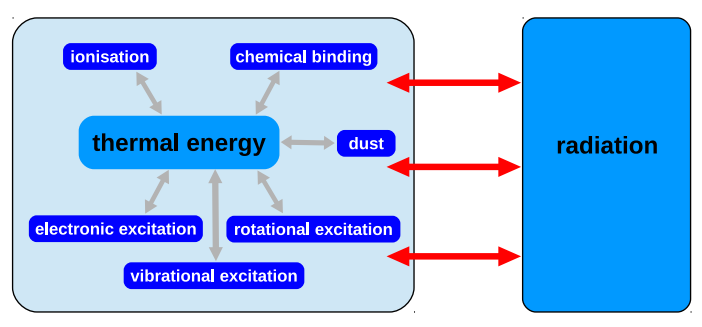

$$
e=\frac{3}{2} n k T_{\mathrm{g}}+E_{\mathrm{rot}}+E_{\mathrm{vib}}+E_{\mathrm{ion}}-E_{\mathrm{diss}}+\ldots
$$

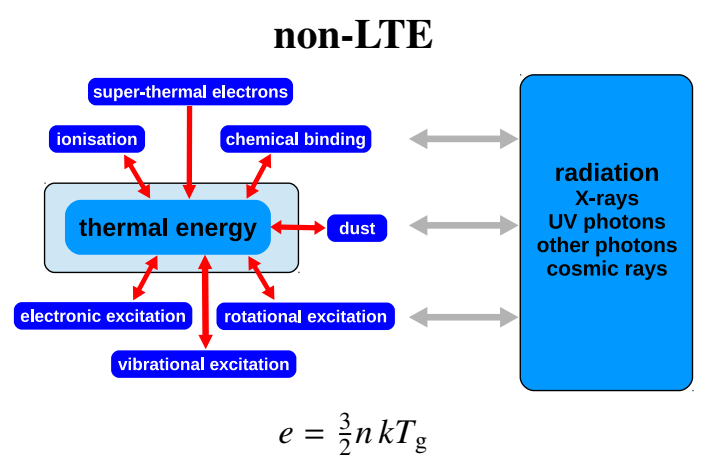

Figure 1. Two approaches for what to include in the internal energy $e$, indicated by the light blue boxes. Following the 1.h.s. LTE approach, $e$ contains all possible ways to store energy in a gas, whereas on the r.h.s. (non-LTE approach) $e$ is simply given by the thermal kinetic energy of the gas particles only. The red arrows visualise energy fluxes considered to calculate the net heating/cooling rate $Q$. In contrast, the grey arrows visualise processes that are not included in the computation of $Q$. 
approach (called "LTE") is mainly used by the stellar interior and stellar atmosphere communities, studying dense gases. Here, the definition of the internal energy comprises all possible ways to store energy in the gas (and dust), including ionisation potentials, chemical binding energies, etc.. Internal processes (mostly of collisional character, grey arrows on the 1.h.s.) are assumed to be fast, redistributing the available energy quickly and evenly among all degrees of freedom. The computation of $Q$ is then based on the energy fluxes that connect the included pools of energy to the outside world, i.e. the red fluxes across the boundaries of the light blue box in Fig. 1.

The second approach (called "non-LTE") is mainly used by the interstellar community studying the energetics of rarefied gases, e.g. under the influence of UV and X-ray radiation fields. Here, the internal energy is simply given by the thermal kinetic energy contained in the 3 translational degrees of freedom of the gas particles. All other forms of energy, like ionisation and dissociation, even rotational, vibrational and electronic excitation, are not expected to be populated thermally anyway (sometimes except for the the rotational degrees of $\mathrm{H}_{2}$ ). The main task then is to compute how these external pools of energy couple to the thermal energy, i.e., again, to calculate the red energy fluxes across the boundaries of the light blue box.

We will follow the non-LTE approach in the remainder of this lecture, but for introduction, a few quite general results of the LTE approach shall be discussed first.

\subsection{Radiative heating/cooling in the LTE case}

In local thermodynamical equilibrium (LTE), the heating/cooling rate is entirely radiative (the outside world are photons as argued above). Assuming LTE, Kirchhoff's law applies $\epsilon_{v}=\kappa_{v}^{\text {abs }} B_{v}(T)$, and balancing the gains and losses of photon energy results in

$$
Q=\iint \kappa_{v}^{\mathrm{abs}} I_{v}(\vec{n}) d \Omega d v-4 \pi \int \epsilon_{v} d v=4 \pi \int \kappa_{v}^{\mathrm{abs}}\left(J_{v}-B_{v}(T)\right) d v,
$$

where $\kappa_{v}^{\text {abs }}[1 / \mathrm{cm}]$ is the gas absorption coefficient at frequency $v[\mathrm{~Hz}], B_{v}(T)\left[\mathrm{erg} / \mathrm{s} / \mathrm{cm}^{2} / \mathrm{Hz} / \mathrm{sr}\right]$ is the Planck function, and $\epsilon_{v}\left[\mathrm{erg} / \mathrm{s} / \mathrm{cm}^{3} / \mathrm{Hz} / \mathrm{sr}\right]$ is the gas emission coefficient. $I_{v}(\vec{n})\left[\mathrm{erg} / \mathrm{s} / \mathrm{cm}^{2} / \mathrm{Hz} / \mathrm{sr}\right]$ is the spectral intensity in direction of the unit vector $\vec{n}, J_{v}=\frac{1}{4 \pi} \int I_{v}(\vec{n}) d \Omega$ is the mean spectral intensity, and $d \Omega[\mathrm{sr}]$ is the 2D solid angle element. Note that $Q$ has units $\left[\mathrm{erg} / \mathrm{cm}^{3} / \mathrm{s}\right]$.

Figure 2 visualises some general findings in LTE. The typical situation (in disks, but not only there) is that the gas is exposed to some distant radiation sources that are intrinsically hotter than the local gas, for example $J_{v} \approx W B_{v}\left(T_{\text {rad }}\right)$ with $W \ll 1$ and $T_{\text {rad }}>T_{\mathrm{g}}$. This applies, in particular, to the optically thin gas above the optical depth $\tau=1$ surface of the disk. Only little of that irradiation penetrates into the optically thick deeper layers, but even there, the typical situation of astrophysical gases is that the local radiation field has a somewhat "bluer" characteristic than the local Planck function. Looking at Eq. (4), this immediately implies the following:

- radiative heating is caused by high-energy absorption processes in the blue part of the spectrum, where $\kappa_{v}^{\mathrm{abs}}$ is large, for example UV-lines of atoms, ions and molecules, or bound-free processes,

- radiative cooling is caused by gas emission lines at long wavelengths, for example atomic finestructure lines, or rotational and ro-vibrational molecular lines.

These conclusions are so basic, that they normally hold even in non-LTE. In the depicted example, the intersection point between $J_{v}$ and $B_{v}$ is located around $\lambda \approx 1.5 \mu \mathrm{m}$. All radiative interactions shortward of that wavelength cause net heating, all other processes cause net cooling. 

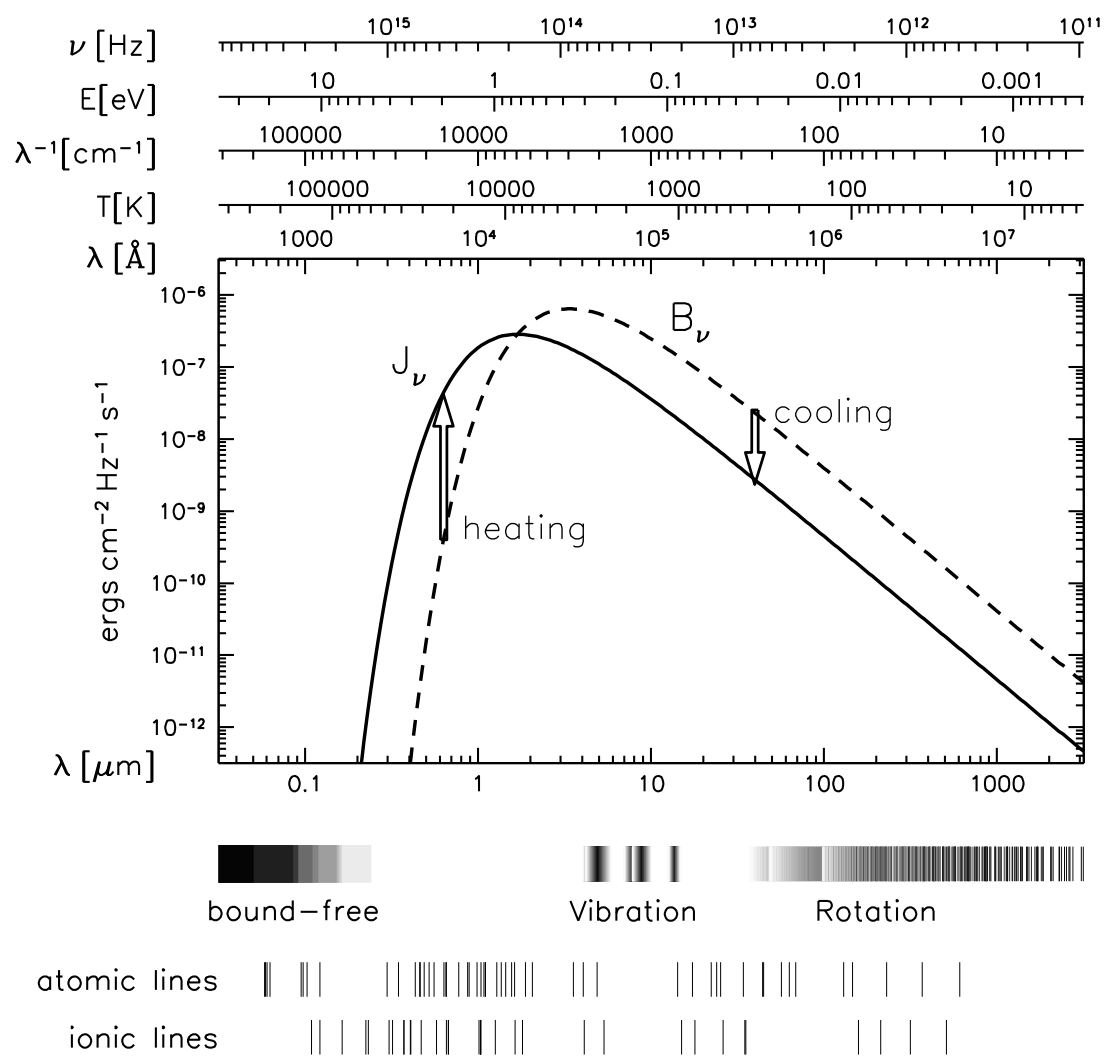

Figure 2. Overview of the radiative heating and cooling in LTE. The full line shows a diluted Planckian $J_{v}=$ $W B_{v}\left(T_{\text {rad }}\right)$ with dilution factor $W=0.029$ and radiation temperature $T_{\text {rad }}=3000 \mathrm{~K}$. The dashed line is the Planck function for $T_{\mathrm{g}}=1500 \mathrm{~K}$. The arrows indicate the energy exchange between matter and radiation, favouring radiative heating at short and radiative cooling at long wavelengths, respectively. The lower panel indicates the wavelength regions of some selected radiative processes. Figure taken from Woitke et al. (1997).

\section{$2 N$-level heating and cooling}

Switching to the non-LTE approach from now on, the most basic interaction between matter and radiation is the absorption and emission of line photons. Let's consider any atomic or molecular system consisting of $N$ discrete energy levels $E_{i}(i=1, \ldots, N)$ [erg]. The first and main task is to compute the non-LTE level populations $n_{i}\left[1 / \mathrm{cm}^{3}\right]$ from the various radiative and collisional rates (Mihalas 1978):

$$
\begin{gathered}
n_{i} \sum_{j \neq i} R_{i j}=\sum_{j \neq i} n_{j} R_{j i} \\
R_{u l}=A_{u l}+B_{u l} \bar{J}_{u l}+C_{u l} \quad, \quad R_{l u}=B_{l u} \bar{J}_{u l}+C_{l u},
\end{gathered}
$$

where $n_{\mathrm{sp}}=\sum_{i} n_{i}$ is the total particle density, $u>l$ denote an upper and lower level $\left(E_{u}>E_{l}\right)$, $A_{u l}[1 / s]$ is the Einstein emission coefficient, $B_{l u}=A_{u l} c^{2} /\left(2 h v_{u l}^{3}\right)$ and $B_{u l}=B_{u l} g_{u} / g_{l}\left[\mathrm{~cm}^{2} \mathrm{~Hz} / \mathrm{erg}\right.$ ] are the Einstein coefficients for absorption and stimulated emission, $C_{u l}=\sum_{k} n_{k} \gamma_{u l}^{k}\left(T_{\mathrm{g}}\right)[1 / \mathrm{s}]$ are the collisional de-excitation rates, $C_{l u}=C_{u l} \frac{g_{u}}{g_{l}} \exp \left(-\Delta E_{u l} / k T_{\mathrm{g}}\right)$ are the collisional excitation rates, $g_{u}, g_{l}$ 
Summer School "Protoplanetary Disks: Theory and Modeling Meet Observations"

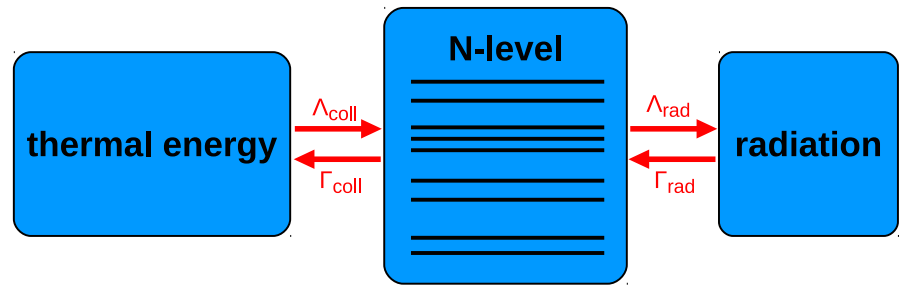

Figure 3. $N$-level heating and cooling rate. Energy fluxes from left to right are cooling, and from right to left heating. Subscript "coll" means collisional, and subscript "rad" means radiative.

are the statistical weights, $n_{k}\left[1 / \mathrm{cm}^{3}\right]$ the collision partner densities, and

$$
\bar{J}_{u l}=\frac{1}{4 \pi} \iint \phi_{u l}(v, \vec{n}) I_{v}(\vec{n}) d v d \Omega
$$

is the line profile averaged mean intensity in units $\left[\mathrm{erg} / \mathrm{cm}^{2} / \mathrm{s} / \mathrm{Hz} / \mathrm{sr}\right]$, where $\phi_{u l}(v, \vec{n})[1 / \mathrm{Hz}]$ is the local line profile function in direction $\vec{n}$. Once the level populations $n_{i}$ have been determined, it is straightforward to compute the net gain of thermal energy. If the $N$-system is closed and timeindependent, as depicted in Fig. 3, we can measure the net heating rate by either balancing the energy fluxes due to the collisional processes, or by balancing the radiative energy fluxes

$$
Q_{N-\text { level }}=\Gamma_{\text {rad }}-\Lambda_{\text {rad }}=\Gamma_{\text {coll }}-\Lambda_{\text {coll }}
$$

The radiative energy fluxes are discussed later. The collisional energy fluxes are given by

$$
\Gamma_{\mathrm{col}}=\sum_{l=1}^{N-1} \sum_{u=l+1}^{N} n_{u} C_{u l} \Delta E_{u l} \quad, \quad \Lambda_{\mathrm{col}}=\sum_{l=1}^{N-1} \sum_{u=l+1}^{N} n_{l} C_{l u} \Delta E_{u l},
$$

where $\Delta E_{u l}=E_{u}-E_{l}$ is the energy difference between level $u$ and $l$. Note that $\Gamma_{\text {col }}-\Lambda_{\text {col }}=0$ in LTE.

The first mayor practical problem is where to get the radiative data from $\left(E_{i}, g_{i}, A_{u l}\right)$, and, in particular, where to get the collisional data from $\left(\gamma_{u l}^{k}\left(T_{\mathrm{g}}\right)\right)$, for all relevant collision partners $k$, for all level combinations $u, l$, and at least for some temperature points that roughly cover the expected gas temperatures in disks. Here, although the data situation has largely improved during the last decades in form of various online platforms, the completeness of the collisional data, in particular for molecules, is still a substantial problem. A good starting point to look for radiative and collisional datasets suitable for astrophysical non-LTE modelling is the Leiden Atomic and Molecular Database, see http://home.strw.leidenuniv.nl/ moldata.

The main physical problem is how to calculate $\bar{J}_{u l}$ for all $u, l$ ? If all $\bar{J}_{u l}$ are known, for example if all lines are optically thin, then Eq. (5) becomes a linear system of equations, and $n_{i}$ can be computed in one go, without any iterations. However, in general, $\bar{J}_{u l}$ depends on the level populations anywhere in the model volume, and an iterative method must be applied. Moreover, calculating $\bar{J}_{u l}$ (Eq. 7) requires to solve the line radiative transfer equation from every spatial grid point, along a sufficient set of rays covering the full solid angle, and for a sufficient number of frequency points to cover the spectral line. For a 2D disk, this would require to solve the line radiative transfer equation $N_{r} \times N_{z} \times N_{\theta} \times N_{\phi} \times N_{v} \approx$ $10^{6} \ldots 10^{8}$ times, where $N_{r}, N_{z}, N_{\theta}, N_{\phi}, N_{v}$ denote the requested numbers of grid points for the radius $r$, the height $z$, the solid angle $(\theta, \phi)$ and the frequency $v$, respectively. And that is true for one spectral line only, and for one iteration step only. To compute the line heating/cooling in disks, we need to consider thousands or rather tens of thousands of lines (just look at $\mathrm{CO}$ ro-vibrational, for example), so a brute force method as sketched above is simply out of the question. 


\subsection{Escape probability}

A simple, beautiful, and closed analytic solution of the above problem can be derived by using a certain set of assumptions called the "escape probability method" (Avrett \& Hummer 1965; Mihalas 1978). It is the key to perform the line heating/cooling computations required in disk models (e.g. Bruderer et al. 2014; Gorti \& Hollenbach 2009; Woitke et al. 2009).

We start with the realisation that, in a disk in Keplerian rotation, there are no local radial velocitygradients along the 6 principal directions (up, down, inward, outward, tangentially forward and backward $)^{1}$, so the escape of line photon in disks can be approximately treated as in a static medium. The first approximation is that we replace the line source functions at any other point along any ray by the local line source function $S_{u l}^{\mathrm{L}}$. Often, the lines are extremely optically thick, so line photons do not travel far, and the main effect we need to take into account is the modification of continuum background radiation with line absorption and emission in a quite small "resonance region" around the considered point. The second approximation is about the geometry of line emission. In a 2D disk, the main escape direction for line photons is vertically upward, so we approximate the geometry by a $1 \mathrm{D}$ semi-infinite slab. Using these assumptions, it is possible to solve the line transfer equation analytically

$$
\begin{aligned}
I_{v}(\mu) & =I_{v}^{0}(\mu) \exp \left(-\tau_{v}^{\mathrm{cont}}(\mu)-\tau_{v}^{\mathrm{L}}(\mu)\right)+\int_{0}^{\tau_{v}^{\text {cont }}(\mu)+\tau_{\nu}^{\mathrm{L}}(\mu)} S_{v}\left(\tau_{v}^{\prime}\right) \exp \left(-\tau_{v}^{\prime}\right) d \tau_{v}^{\prime} \\
& \approx I_{v_{u l}}^{\text {cont }}(\mu) \exp \left(-\tau_{v}^{\mathrm{L}}(\mu)\right)+\int_{0}^{\tau_{v}^{\mathrm{L}}(\mu)} S_{u l}^{\mathrm{L}}\left(\tau_{v}^{\prime}\right) \exp \left(-\tau_{v}^{\prime}\right) d \tau_{v}^{\prime} \\
& \approx I_{v_{u l}}^{\mathrm{cont}}(\mu) \exp \left(-\tau_{v}^{\mathrm{L}}(\mu)\right)+S_{u l}^{\mathrm{L}}\left(1-\exp \left(-\tau_{v}^{\mathrm{L}}(\mu)\right)\right) \\
S_{u l}^{\mathrm{L}} & =\frac{2 h v_{u l}^{3}}{c^{2}}\left(\frac{g_{u} n_{l}}{g_{l} n_{u}}-1\right)^{-1},
\end{aligned}
$$

where $I_{v}^{0}(\mu)$ is the irradiation from the boundary, $S_{v}$ is the general line + continuum source function, $S_{u l}^{\mathrm{L}}\left[\mathrm{erg} / \mathrm{cm}^{2} / \mathrm{s} / \mathrm{Hz} / \mathrm{sr}\right]$ is the local line source function, $\tau_{v}^{\text {cont }}(\mu)$ is the continuum optical depth and $\tau_{v}^{\mathrm{L}}(\mu)$ the line optical depth, measured from the boundary to the point of interest. $I_{v_{u l}}^{\text {cont }}(\mu)$ is the local background continuous intensity at line centre frequency $v_{u l}$, the result of a continuum radiative transfer for $I_{v}(\mu)$ if line emission and absorption in the considered spectral line are disregarded.

To compute $\bar{J}_{u l}$ according to Eq. (7), we use Eq. (12) for all directions $\mu=\cos (\theta)$. For the first term in Eq. (12) we assume that the continuous intensities mainly originate from the central star directly, so we use the radial line optical depth $\tau_{v}^{\mathrm{L}}=\tau_{u l}^{\mathrm{rad}} \phi(x)$. For the second term, since the line emission is isotropic, we use the assumption of slab geometry in $z$-direction $\tau_{v}^{\mathrm{L}}(\mu)=\tau_{u l}^{\mathrm{ver}} \phi(x) / \mu$, introducing a dimensionless line profile function of Doppler type $\phi(x)=\exp \left(-x^{2}\right) / \sqrt{\pi}$, where $x=\left(v-v_{u l}\right) / \Delta v_{D}$. The frequency width $\Delta v_{D}=v_{u l} \Delta \mathrm{v}_{D} / c$ is given by the velocity width $\Delta \mathrm{v}_{D}=\sqrt{\frac{2 k T_{\mathrm{gas}}}{m_{\mathrm{sp}}}+v_{\mathrm{turb}}^{2}}$, where $m_{\mathrm{sp}}$ is the species mass and $v_{\text {turb }}$ the turbulent velocity. The result is

$$
\begin{aligned}
\bar{J}_{u l} & \approx P_{u l}^{\mathrm{pump}} J_{v_{u l}}^{\mathrm{cont}}+\left(1-P_{u l}^{\mathrm{esc}}\right) S_{u l}^{\mathrm{L}} \\
P_{u l}^{\mathrm{pump}}\left(\tau_{u l}^{\mathrm{rad}}\right) & =\int_{-\infty}^{+\infty} \phi(x) \exp \left(-\tau_{u l}^{\mathrm{rad}} \phi(x)\right) d x \\
P_{u l}^{\mathrm{esc}}\left(\tau_{u l}^{\mathrm{ver}}\right) & =\frac{1}{2} \int_{-\infty}^{+\infty} \phi(x) \int_{0}^{1} \exp \left(-\frac{\tau_{u l}^{\mathrm{ver}} \phi(x)}{\mu}\right) d \mu d x
\end{aligned}
$$

\footnotetext{
${ }^{1}$ Although there are actually local non-zero velocity gradients in all other directions!
} 


$$
\begin{aligned}
\tau_{u l}^{\mathrm{rad}} & =\int_{0}^{r} \frac{A_{u l} c^{3}}{8 \pi v_{u l}^{3} \Delta \mathrm{v}_{D}}\left(n_{l}\left(r^{\prime}\right) \frac{g_{u}}{g_{l}}-n_{u}\left(r^{\prime}\right)\right) d r^{\prime} \\
\tau_{u l}^{\mathrm{ver}} & =\int_{z}^{\infty} \frac{A_{u l} c^{3}}{8 \pi v_{u l}^{3} \Delta \mathrm{v}_{D}}\left(n_{l}\left(z^{\prime}\right) \frac{g_{u}}{g_{l}}-n_{u}\left(z^{\prime}\right)\right) d z^{\prime},
\end{aligned}
$$

where $P_{u l}^{\text {esc }}$ is the escape probability and $P_{u l}^{\text {pump }}$ the pumping probability, which only depends on line optical depth, and can be pre-tabulated, or treated with approximate formulae (Woitke et al. 2009). $\tau_{u l}^{\mathrm{rad}}$ and $\tau_{u l}^{\mathrm{ver}}$ are the radial and vertical line optical depths.

Equation (14) is the central equation of the escape probability method. If the line is optically thin, $\bar{J}_{u l}$ is simply given by $J_{v_{u l}}^{\text {cont }}$, i.e. the result from a pure continuum radiative transfer. If the line optical depths become large, $P_{u l}^{\text {esc }}$ and $P_{u l}^{\text {pump }}$ become small, and $J_{v_{u l}}^{\text {cont }}$ is more and more replaced by the local line source function. In other words, an optically thick line sees its own source function. The solution of the non-LTE rate equations (Eq. 5) becomes very easy this way. In fact, one can eliminate the unknown line source function $S_{u l}^{\mathrm{L}}$ by introducing the following effective rates $\tilde{R}_{u l}=$ $A_{u l} P_{u l}^{\text {esc }}+B_{u l} P_{u l}^{\text {pump }} J_{v_{u l}}^{\text {cont }}+C_{u l}$ and $\tilde{R}_{l u}=B_{l u} P_{u l}^{\text {pump }} J_{v_{u l}}^{\text {cont }}+C_{l u}$. This procedure is very advantageous numerically, and dramatically improves convergence and stability in optically thick regions, because it avoids to trace any emitted line photons that are directly re-absorbed locally, which has no net effect on the level populations.

The derivation of Eq. (14) shows, however, that the 2D disk geometry raises a few cumbersome questions about what are the appropriate paths or directions of continuous photons making their way to the point of interest, and how line photons escape from that point. Since the irradiation mainly comes from a point source (the star), but the emission is isotropic, the corresponding pumping and escape probabilities will not be the same in $2 \mathrm{D}$ or $3 \mathrm{D} P_{u l}^{\text {pump }} \neq P_{u l}^{\text {esc }}$. This problem is similar to finding appropriate "self-shielding" factors for the reduction of photo-ionisation and photodissociation rates in chemistry.

Another principle problem of the escape probability method is that it doesn't take into account the effect of distant strong line source functions. In protoplanetary disks, there is often a disk layer where the densities are high, so the level populations are close to LTE, i.e. strong, and a lot of line photons are emitted from the top of that layer into the optically thin model volume above. In these tenuous layers, we get non-LTE populations in the optically thin case, but the recipe Eq. (14) tends to underpredict the level populations, because it uses the local line source function only, which is low because it's radiatively de-populated. Further discussions about the pumping and escape probabilities in discs can be found in (Woitke et al. 2009, see their Fig. 5 and text on page 393).

Kamp et al. (2010, see their Fig. 6), and also Kamp (2015) have made detailed comparisons between the results from escape probability and from the detailed Monte-Carlo line radiative transfer code RATRAN (Hogerheijde \& van der Tak 2000). In case of the 3 fine-structure lines of OI, they found very good agreement between the predicted line fluxes (deviation $<5 \%$ ) whereas assuming LTE would overpredict the lines by up to $70 \%$. This is a fine example of an un-written law in non-LTE line transfer, namely that the results from escape probability are often surprisingly accurate, despite the strong assumptions used.

\subsection{Computation of the net heating rate}

The dependency of $P_{u l}^{\text {esc }}$ and $P_{u l}^{\text {pump }}$ on the respective line optical depths introduces a further complication. The last increments of the radial and vertical line optical depths $\Delta \tau_{u l}^{\mathrm{rad}}$ and $\Delta \tau_{u l}^{\mathrm{ver}}$, from the neighbouring grid points to the current grid point, depend on the level populations in the current point, which we wish to compute. 
This dependency requires to use an iterative approach, and we use a fully implicit integration scheme where the $\Delta \tau$ are calculated according to the spatial path lengths between these points $\times$ the local level populations. The usage of such an implicit scheme seems necessary to stabilise the line optical depth integrations. In order to check the quality of solution, and convergence of the implicit $\tau$ integration scheme, we compute the radiative energy fluxes as

$$
\begin{aligned}
\Gamma_{\mathrm{rad}} & =\sum_{\text {lines }} n_{l} \Delta E_{u l} P_{u l}^{\mathrm{pump}} B_{l u} J_{v_{u l}}^{\mathrm{cont}} \\
\Lambda_{\mathrm{rad}} & =\sum_{\text {lines }} n_{u} \Delta E_{u l}\left(P_{u l}^{\mathrm{esc}} A_{u l}+P_{u l}^{\mathrm{pump}} B_{u l} J_{v_{u l} \mathrm{cont}}\right)
\end{aligned}
$$

and use Eq. (8) to check whether indeed $\Gamma_{\text {rad }}-\Lambda_{\text {rad }}=\Gamma_{\text {coll }}-\Lambda_{\text {coll }}$ is valid.

Equations (19) and (20) shows again how the various chemical and physical processes are interlinked in disks. In order to calculate the line heating/cooling rates, we need to know the various particle and collision partner densities $n_{\mathrm{sp}}, n_{k}$ ( $\rightarrow$ chemistry), and we need to know the continuous background radiation field $J_{v_{u l}}^{\text {cont }}(\rightarrow$ continuum radiative transfer). Vice versa, the chemistry requires the gas and dust temperatures ( $\rightarrow$ heating \& cooling, $\rightarrow$ continuum radiative transfer).

Figure 4 shows the resulting line cooling rate $Q$ for one exemplary atomic fine-structure line (left) and for $\mathrm{CO}$ ro-vibrational cooling (right), without continuous background radiation (from Woitke et al. 1996). Three different line cooling regimes can be distinguished depending on the relation between the local gas density $n_{\langle\mathrm{H}\rangle}$, the critical density for LTE population $n_{\mathrm{cr}}$, and the density where the considered line becomes optically thick $n_{\text {thick }}$. The case discussed in Fig. 4 is different from the static escape probability method applied to protoplanetary disks (Eqs. 17 and 18), namely the limiting case of large velocity gradients $\left\langle\frac{d v}{d l}\right\rangle$ (Sobolev theory) in a spherical medium, but the concept and
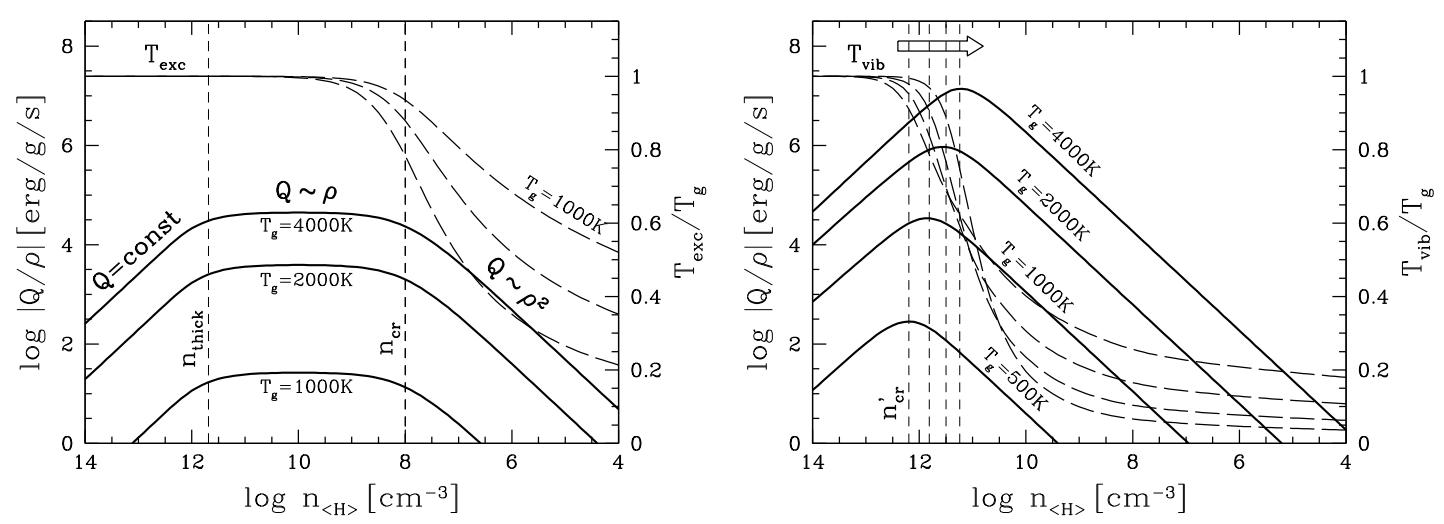

Figure 4. Example atomic fine-structure line cooling rate per mass $Q / \rho$ (left), and $\mathrm{CO}$ vibrational cooling (right), from Woitke et al. (1996, reproduced with permission (C) ESO). $J_{v}^{\text {cont }}=0$ is assumed here, and a different way to compute the escape probabilities for an expanding gas in spherical symmetry is applied (Sobolev limit of large local velocity gradients). 
fundamental results are comparable. In this limiting case, the critical densities are given by

$$
\begin{aligned}
n_{\mathrm{cr}} & =\frac{A_{u l}}{C_{u l} / n_{<\mathrm{H}>}} \\
n_{\text {thick }} & =\frac{8 \pi}{A_{u l}} \frac{g_{l}}{g_{u}}\left(\frac{v_{u l}}{c}\right)^{3}\left\langle\frac{d v}{d l}\right\rangle \frac{n_{<\mathrm{H}>}}{n_{\mathrm{sp}}} \\
\left\langle\frac{d v}{d l}\right\rangle & =\frac{1}{3}\left|\frac{\partial v}{\partial r}\right|+\frac{2}{3}\left(2 x_{0}^{-1 / 2}-1\right)\left|\frac{v}{r}\right| \quad \text { where } \quad x_{0}=1+\max \left\{0,-\frac{\partial v}{\partial r} \mid \frac{v}{r}\right\},
\end{aligned}
$$

see further details in (Woitke et al. 1996). At low densities $n_{\langle\mathrm{H}\rangle}<n_{\mathrm{cr}}$, the cooling rate scales as $Q \propto \rho^{2}$ and the levels are populated sub-thermally $T_{\text {exc }}<T_{\mathrm{g}}$. At very high densities $n_{\langle\mathrm{H}\rangle}>n_{\text {thick }}$, all lines are optically thick, the levels are thermally populated, and $Q$ becomes constant, proportional to the number of considered spectral lines. In between, we have the case of optically thin LTE cooling, where the cooling rate/gas mass is most efficient and scales as $Q \propto \rho$. In case of CO ro-vibrational cooling, which is a superposition of hundreds of individual lines, we find $n_{\mathrm{cr}}>n_{\text {thick}}$. Here, optically thin LTE cooling never occurs, but the cooling behaviour changes directly from $Q=$ const to $Q \propto \rho^{2}$ at $n_{\mathrm{cr}}^{\prime}=\sqrt{n_{\mathrm{cr}} n_{\text {thick }}}$. The ro-vibrational levels are thermally populated already at $n_{\langle\mathrm{H}\rangle}>n_{\mathrm{cr}}^{\prime}\left(n_{\mathrm{cr}}^{\prime}<n_{\mathrm{cr}}\right)$.

\section{Photo-ionisation heating and recombination cooling}

Consider a pair of photo-processes of type

$$
\mathrm{AB}+h v \underset{k_{r}}{\stackrel{k_{f}}{\rightleftharpoons}} \mathrm{A}+\mathrm{B} .
$$

Applications in mind could be the photo-ionisation and direct recombination of $\mathrm{H}^{-}\left(\mathrm{AB}=\mathrm{H}^{-}, \mathrm{A}=\mathrm{H}\right.$, $\left.\mathrm{B}=\mathrm{e}^{-}\right)$, or the bound-free photo-ionisation of neutral carbon $\left(\mathrm{AB}=\mathrm{C}, \mathrm{A}=\mathrm{C}^{+}, \mathrm{B}=\mathrm{e}^{-}\right.$), but the following method may also be applicable to a more general pair of photo-dissociation and radiative association reactions. The forward rate coefficient $k_{\mathrm{f}}[1 / \mathrm{s}]$ is given by

$$
k_{f}=4 \pi \int_{v_{\text {thr }}}^{\infty} \frac{J_{v}}{h v} \sigma^{\mathrm{bf}}(v) d v,
$$

such that $n_{\mathrm{AB}} k_{f}\left[\mathrm{~cm}^{-3} \mathrm{~s}^{-1}\right]$ is the number of photo-ionisations per volume and time. $\sigma^{\mathrm{bf}}(v)$ is the photo cross section $\left[\mathrm{cm}^{2}\right]$ of $\mathrm{AB}$ for the bound-free forward reaction. Energy conservation implies that

$$
h v=E_{b}+E_{\mathrm{th}}
$$

where $E_{b}=h v_{\text {thr }}$ is the binding energy of $\mathrm{AB}, v_{\mathrm{thr}}$ is the threshold frequency, and $E_{\mathrm{th}}=\frac{1}{2} m v^{2}$ is the excess thermal energy liberated by the photo-reaction. Note that, in general, A and B could also end up in an electronically excited state, or, if A or B are molecules, they could carry away rotational and vibrational excitation energies as well. Using Eq. (26) would then assume that all these excitation energies are also thermalised in some way. The bound-free photo-ionisation heating rate $\Gamma_{\mathrm{bf}}\left[\mathrm{erg} \mathrm{s}^{-1} \mathrm{~cm}^{-3}\right]$ is then given by

$$
\Gamma_{\mathrm{bf}}=n_{\mathrm{AB}} 4 \pi \int_{v_{\mathrm{thr}}}^{\infty} J_{v} \frac{v-v_{\mathrm{thr}}}{v} \sigma^{\mathrm{bf}}(v) d v .
$$


The reverse process, the recombination cooling rate, is slightly more difficult to understand. A direct recombination, or radiative association, reduces the number of particles by one (e.g. one thermal electron disappears), so the cooling rate should be of order $n_{\mathrm{A}} n_{\mathrm{B}} k_{r}\left(T_{\mathrm{g}}\right)\left\langle E_{\mathrm{th}}\right\rangle$, where the mean disappearing thermal energy $\left\langle E_{\mathrm{th}}\right\rangle \approx k T_{\mathrm{g}}$. Let's assume that the recombination rate coefficient is given by an Arrhenius law with fit coefficients $\alpha, \beta, \gamma$ as

$$
k_{r}\left(T_{\mathrm{g}}\right)=\alpha\left(\frac{T_{\mathrm{g}}}{300 \mathrm{~K}}\right)^{\beta} \exp \left(-\frac{\gamma}{T_{\mathrm{g}}}\right) .
$$

In order to investigate the details of $\left\langle E_{\mathrm{th}}\right\rangle$, we consider the pair of photo-processes (24) in the case of thermodynamical equilibrium, where $J_{v}=B_{v}\left(T_{\mathrm{g}}\right)$ and where every reaction is in detailed balance with its direct reverse, i.e.

$$
\left.n_{\mathrm{AB}}^{\star} k_{f}\right|_{J_{v}=B_{v}\left(T_{\mathrm{g}}\right)}=n_{\mathrm{A}}^{\star} n_{\mathrm{B}}^{\star} k_{r}\left(T_{\mathrm{g}}\right) .
$$

where the ${ }^{\star}$ superscript indicates the particle densities in thermodynamical equilibrium. Thus, we find the recombination rate coefficient to be

$$
k_{r}\left(T_{\mathrm{g}}\right)=\frac{n_{\mathrm{AB}}^{\star}}{n_{\mathrm{A}}^{\star} n_{\mathrm{B}}^{\star}} 4 \pi \int_{v_{\mathrm{thr}}}^{\infty} \frac{B_{v}\left(T_{\mathrm{g}}\right)}{h v} \sigma^{\mathrm{bf}}(v) d v,
$$

which is known as the bound-free Milne relation (Mihalas 1978). Equation (30) allows us to calculate the recombination cooling rate $\Lambda_{\mathrm{bf}}\left[\mathrm{erg} \mathrm{s}^{-1} \mathrm{~cm}^{-3}\right]$ analogous to Eq. (27)

$$
\Lambda_{\mathrm{bf}}=n_{\mathrm{A}} n_{\mathrm{B}} \frac{n_{\mathrm{AB}}^{\star}}{n_{\mathrm{A}}^{\star} n_{\mathrm{B}}^{\star}} 4 \pi \int_{v_{\mathrm{thr}}}^{\infty} B_{v}\left(T_{\mathrm{g}}\right) \frac{v-v_{\mathrm{thr}}}{v} \sigma^{\mathrm{bf}}(v) d v .
$$

Finally, we eliminate the fraction $n_{\mathrm{AB}}^{\star} /\left(n_{\mathrm{A}}^{\star} n_{\mathrm{B}}^{\star}\right)$ by using Eqs. (28) and (30)

$$
\Lambda_{\mathrm{bf}}=n_{\mathrm{A}} n_{\mathrm{B}} k_{r}\left(T_{\mathrm{g}}\right) \frac{\int_{v_{\mathrm{thr}}}^{\infty} B_{v}\left(T_{\mathrm{g}}\right) \frac{v-v_{\mathrm{thr}}}{v} \sigma^{\mathrm{bf}}(v) d v}{\int_{v_{\mathrm{thr}}}^{\infty} \frac{B_{v}\left(T_{\mathrm{g}}\right)}{h v} \sigma^{\mathrm{bf}}(v) d v},
$$

where the r.h.s. fraction can be identified as the mean thermal energy $\left\langle E_{\mathrm{th}}\right\rangle$ disappearing from the gas phase due to a recombination process.

\section{Free-free transitions}

Collisions between electrons and other particles lead to sudden accelerations of the electrons during the encounter which cause the emission of radiation (Bremsstrahlung). These free-free transitions directly convert thermal energy into photon energy (free-free cooling). The reverse process is a 3body encounter of an electron with another gas particle and a photon, which can destroy the photon and lead an acceleration of the electron (free-free-heating). Since these processes are completely controlled by the thermal motion of the charged particles, we can assume LTE and apply Eq. (4). The heating rate $\Gamma_{\mathrm{ff}}$ and cooling rate $\Lambda_{\mathrm{ff}}$ are given by

$$
\begin{aligned}
\Gamma_{\mathrm{ff}} & =4 \pi \int \kappa_{v}^{\mathrm{ff}} J_{v} d v \\
\Lambda_{\mathrm{ff}} & =4 \pi \int \kappa_{v}^{\mathrm{ff}} B_{v}\left(T_{\mathrm{g}}\right) d v \\
\kappa_{v}^{\mathrm{ff}} & =n_{\mathrm{e}}^{2} \sigma_{v}^{\mathrm{ff}}+n_{\mathrm{e}} n_{\mathrm{H}} \sigma_{v}^{\mathrm{H}^{-} \mathrm{ff}}+n_{\mathrm{e}} n_{\mathrm{H}_{2}} \sigma_{v}^{\mathrm{H}_{2}^{-} \mathrm{ff}}+n_{\mathrm{e}} n_{\mathrm{He}} \sigma_{v}^{\mathrm{He}^{-} \mathrm{ff}},
\end{aligned}
$$




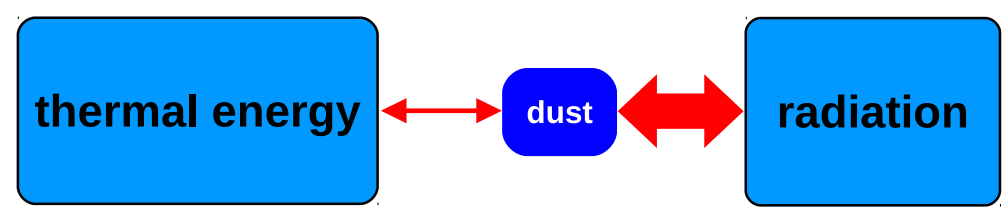

Figure 5. Inelastic collisions between gas and dust (thermal accommodation) lead to an energy exchange between the gas and the dust phases, whereas the dust is usually close to radiative equilibrium, i.e. tightly coupled to the mean energy density in the surrounding radiation field. Any excess dust energy, i.e. received by inelastic gas collisions, is quickly radiated away.

where $\kappa_{v}^{\mathrm{ff}}$ is the free-free gas opacity $\left[\mathrm{cm}^{-1}\right]$. The free-free cross-sections $\sigma_{v}^{\mathrm{ff}}\left[\mathrm{cm}^{5}\right]$ for bremsstrahlung of singly ionised gases can be e.g. taken from (Hummer 1988), for $\mathrm{H}^{-}$ff from (Stilley \& Callaway 1970), for $\mathrm{H}_{2}^{-}$ff from Somerville (1964), and for $\mathrm{He}^{-}$ff from John (1994).

\section{Dust thermal accommodation}

Inelastic collisions lead to an energy exchange between the gas and the dust particles as sketched in Fig. 5. In the microscopic picture, the impinging gas particles (with internal energy $\propto T_{\mathrm{g}}$ ) may get temporarily physisorbed on the surface, where they hop around and accommodate their internal energy with the dust surface $\left(\propto T_{\mathrm{d}}\right)$, before they are released again back into the gas phase. The net heating rate of thermal accommodation can be expressed as

$$
\begin{aligned}
\Gamma_{\mathrm{acc}}-\Lambda_{\mathrm{acc}} & =\pi\left\langle a^{2}\right\rangle n_{d} \sqrt{\frac{8 k T_{\mathrm{g}}}{\pi m_{\mathrm{H}}}} n_{\langle\mathrm{H}\rangle} \alpha_{\mathrm{acc}}\left(2 k T_{\mathrm{d}}-2 k T_{\mathrm{g}}\right) \\
& \approx 4 \times 10^{-12} \pi\left\langle a^{2}\right\rangle n_{d} n_{\langle\mathrm{H}\rangle} \alpha_{\mathrm{acc}} \sqrt{T_{\mathrm{g}}}\left(T_{\mathrm{d}}-T_{\mathrm{g}}\right)
\end{aligned}
$$

(Burke \& Hollenbach 1983), where $\pi\left\langle a^{2}\right\rangle n_{d}$ is the total dust cross section per volume, $a$ the dust particle radius, $\sqrt{8 k T_{\mathrm{g}} /\left(\pi m_{\mathrm{H}}\right)}$ the mean velocity of gas particles, $n_{\langle\mathrm{H}\rangle}$ the gas particle density, and $\left(T_{\mathrm{d}}-T_{\mathrm{g}}\right)$ is proportional to the net energy transfer rate due to inelastic collisions. The efficiency for inelastic collisions is quite high (see Groenewegen 1994)

$$
\alpha_{\mathrm{acc}} \approx 0.1+0.35 \exp \left(-\sqrt{\frac{T_{\mathrm{g}}+T_{\mathrm{d}}}{500 \mathrm{~K}}}\right) .
$$

Groenewegen (1994) fitted the data in Burke \& Hollenbach (1983) using the function form introduced in Hollenbach \& McKee (1979). Due to the $\rho^{2}$-dependency, thermal accommodation usually provides both the dominating heating process and the dominating cooling process for the gas in the disk midplane, as soon as high-energy photons are sufficiently absorbed, enforcing $T_{\mathrm{g}} \approx T_{\mathrm{d}}$ in the midplane.

The influence of the thermal accommodation heating/cooling on the dust energy balance can usually be neglected in models, because the absorption cross sections of dust particles are huge, making sure that the dust temperature $T_{\mathrm{d}}$ remains well coupled to the ambient radiation field. This situation (see Fig. 5) usually turns the thermal accommodation heating/cooling into a small perturbation for the dust energy balance, whereas it remains important for the gas energy balance. However, disks may become massively optically thick in the continuum, in which case the radiation field will quickly adapt to any dust temperature structure. A meaningful example here is viscous heating, which may cause a strong heating of the gas phase in the disk midplane. This heat is then mostly transferred to the 


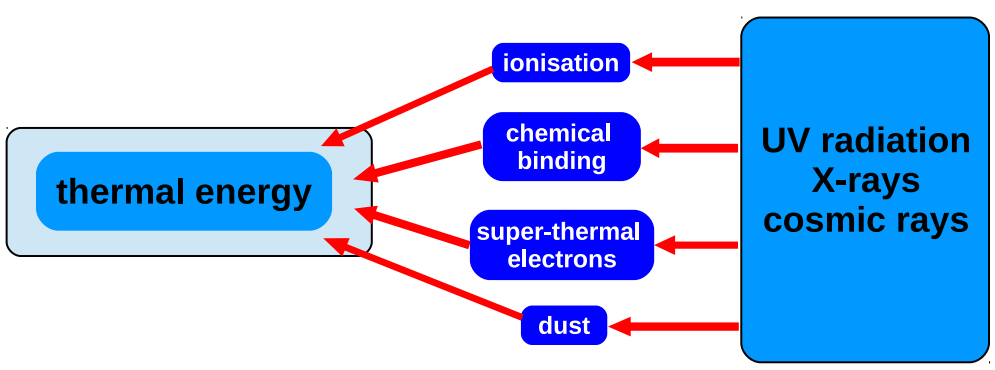

Figure 6. Heating due to high-energy irradiation, triggered by UV, and X-ray photons, and by cosmic rays.

optically thick dust, for example by thermal accommodation, and the dust will then transport that excess heat away, in a diffusive way, toward the disk surface, where it is radiated away. If we want to get the physics of viscous heating correct, it is obviously important to treat the thermal accommodation heating/cooling properly, also in the dust energy balance.

\section{High-energy heating processes}

The central stars of protoplanetary disks are known to be strong UV and X-ray emitters, due to a wealth of high-energy processes occurring in or very close to the stellar surface, usually denoted as "stellar activity" (e.g. chromospheres, coronae, star spots, magnetic reconnections, ...), as well as the final deceleration of the in-falling matter in the accretion columns in form of standing shock fronts. All these complicated processes are expected to occur within a fraction of one, or maybe a few, stellar radii, i.e. well inside the inner rim of the dusty disk, $R_{\star} \approx 2 R_{\odot} \approx 0.01 \mathrm{AU} \ll R_{\text {in }}$. Therefore, in a disk model, we treat these processes as an additional, external (point) source for irradiation of the disk, e.g. with measured spectral flux. The additional, non-photospheric UV and X-ray irradiation causes strong non-LTE effects in the disk, and strong heating of the disk surface layer, just where the gas emission lines come from.

That being said, an important question in this context is how deep UV and X-rays penetrate into the disk, where the assumed position and spatial extension of the UV and X-ray source (see Sect. 6.4), and the geometry of the disk play a crucial role. In the following subsections, we will assume that an UV and X-ray continuum radiative transfer has been carried out (including scattering) such that the local mean intensity $J_{v}$ at UV wavelengths and the local primary X-ray ionisation rate is known.

The energetic effect of the high-energy irradiation (UV, X-rays, also cosmic rays) is clearly one way, pure heating, because the temperatures in the disk are much too low to cause any significant emission at these wavelengths. Without the high-energy heating processes, the molecular gas would actually be expected to be cooler than the dust (see Fig. 2), because the dust, in general, has a "blue" absorption characteristic (see Min 2015), whereas a molecular gas has its majority of spectral lines in the mid-IR and sub-mm which emphasise the cooling. However, if $T_{\mathrm{g}}<T_{\mathrm{d}}$ was true, the disk would be expected to show a wealth of molecular absorption lines in the near and mid-IR where the disk is optically thick. The observational fact that, in contrast, disks generally exhibit gas emission lines, from near-IR to $\mathrm{mm}$ wavelengths, shows that high-energy heating processes must be the key to understand the gas temperatures in disks. 


\subsection{X-ray and cosmic ray heating}

The interaction of X-rays with the gas in a protoplanetary disk (or with cosmic ray particles, which is actually quite similar) involves a cascade of collisional, ionisation, chemical and radiative processes where, step by step, the kinetic energy of the involved photons, ions and electrons is reduced by inelastic processes, until their remaining excess kinetic energy can be thermalised (Glassgold et al. 2012). X-rays start to interact with the gas via primary ionisations, for example kicking out $K$-shell electrons from the ambient atoms and molecules (see Güdel 2015). These super-thermal electrons then keep on speeding through the gas, ionising further particles on their way (secondary ionisations), the ionised particles can produce secondary X-rays (Auger effect), and the recombination of the ionised atoms and molecules produce secondary UV photons which lead to further ionisations.

Detailed studies of this energy cascade have been carried out by Dalgarno et al. (1999). The most effective heating process has been identified to be the Coulomb heating, where the super-thermal electrons undergo elastic collisions with other, thermal electrons which accelerate the latter, i.e. heat the gas. The results are complicated, but can be generally formulated as

$$
\Gamma_{\text {Xray }(\mathrm{Cou})}=\zeta_{\text {Xray }}\left(Q_{\mathrm{H}}^{\mathrm{Cou}} n_{\mathrm{H}}+Q_{\mathrm{H}_{2}}^{\mathrm{Cou}} n_{\mathrm{H}_{2}}+\ldots\right) .
$$

Analogously, the heating of the gas due to cosmic rays is

$$
\Gamma_{\mathrm{CR}}=\zeta_{\mathrm{CR}}\left(Q_{\mathrm{H}}^{\mathrm{CR}} n_{\mathrm{H}}+Q_{\mathrm{H}_{2}}^{\mathrm{CR}} n_{\mathrm{H}_{2}}+\ldots\right),
$$

(see also Hollenbach $\&$ McKee 1989), where $n_{\mathrm{H}}, n_{\mathrm{H}_{2}}$ are the various particle densities, and $\zeta$ Xray, $\zeta_{\mathrm{CR}}[1 / \mathrm{s}]$ are the local primary ionisation (ion-pair production) rates. Equations (38) and (39) are simplified here, adapted to the case of cosmic ray heating. The various $Q$ 's [erg] measure the amount of energy thermalised via Coulomb interactions along the cascade, which is featured by certain branching ratios depending on the initial state of the gas, for example the initial $\mathrm{H} / \mathrm{H}_{2}$ ratio, and the initial degree of ionisation. Typical values for the $Q$ 's range from a fraction to a couple of $\mathrm{eV}$ per ion-pair (Dalgarno et al. 1999).

Cosmic rays (CR) have finite penetration depths, depending on their energy. After convolution with the cosmic ray's initial energy spectrum, the resulting cosmic ray ionisation rate approximately becomes an exponential $\zeta_{\mathrm{CR}}=\zeta_{\mathrm{CR}, 0} \exp \left(-\Sigma / \Sigma_{\mathrm{CR}}\right)$ as function of gas column density $\Sigma\left[\mathrm{g} / \mathrm{cm}^{2}\right]$ (Fujii et al. 2011). However, the value of $\Sigma_{\mathrm{CR}}$ is pretty uncertain, because the initial cosmic ray energy spectrum outside of the heliosphere (i.e. also outside of the Earth's magnetosphere) is not wellknown. Umebayashi \& Nakano (2009) provide a value of $\Sigma_{\mathrm{CR}} \approx 96 \mathrm{~g} / \mathrm{cm}^{2}$, which corresponds to an e-folding hydrogen nuclei column density of about $4 \times 10^{25} \mathrm{~cm}^{-2}$. Whether such magnetic shielding effects might also reduce the cosmic ray ionisation rates in protoplanetary disks is not clear. Cleeves et al. $(2013,2015)$ argue that stellar winds can power a heliosphere-like analog where the cosmic rays are efficiently "absorbed" by the stellar wind plasma which is magnetised, diminishing the CR disk ionisation rates by several orders of magnitude for $\mathrm{CR}$ energies $\lesssim 1 \mathrm{GeV}$.

The unshielded $\mathrm{H}_{2} \mathrm{CR}$ ionisation rate is of order $\zeta_{\mathrm{CR}, 0} \approx 1.4 \times 10^{-17} \mathrm{~s}^{-1}$ (McElroy et al. 2013). However, if the protoplanetary disk under consideration is situated in a star formation cluster which has already developed massive energetic wind bubbles or supernovae, which are likely to produce new cosmic rays in shocks, $\zeta_{\mathrm{CR}, 0}$ might be much larger. In contrast, if magnetic fields can effectively shield the disk, the radioactive decay of primordial ${ }^{26} \mathrm{Al}$ provides a minimum ionisation rate of about $7.3 \times 10^{-19} \mathrm{~s}^{-1}$ (Umebayashi \& Nakano 2009).

\subsection{Dust photoelectric heating}

The photoelectric effect (PE) on dust grains is known to provide one of the major heating mechanism for the interstellar medium (ISM), see e.g. Bakes \& Tielens (1994). Absorption of UV radiation by 
a dust grain can lead to the ejection of a non-thermal electron (and a positive charging of the grain) beyond a certain threshold energy according to

$$
h v=\frac{1}{2} m_{\mathrm{e}} v_{\mathrm{e}}^{2}+e U
$$

where $h v$ is the energy of the UV photon, $e U$ is the work function at the surface of the grain, i.e. the minimum energy required to remove a delocalised electron from the surface, which depends on size, charge, and material of the grain. The excess kinetic energy $\frac{1}{2} m_{\mathrm{e}} v_{\mathrm{e}}^{2}$ of the escaping electron is assumed to be thermalised.

In order to calculate the total PE heating rate, we must solve consistently the charging balance of the grains. The PE leads to positive grain charges, but collisions with gaseous ions and electrons tend to charge the grains negatively, because the thermal velocities of free electrons are larger than the thermal velocities of positive ions. In principle, the different charged states of dust grains must be included in the chemistry to get consistent results including the electron concentration, which is effected by the grain charging. Detailed studies have been done under conditions typical for the interstellar medium (e.g. Bakes \& Tielens 1994). The result can be summarised as

$$
\Gamma_{\mathrm{PE}}=2.5 \times 10^{-4} \epsilon(\mathrm{x}) \kappa_{\mathrm{dust}}^{\mathrm{abs}} \chi
$$

where $\epsilon$ is a material-dependent efficiency that depends on grain charge parameter $\mathrm{x}=\sqrt{T_{\mathrm{gas}}} \chi / n_{\mathrm{el}}$, where $n_{\mathrm{el}}\left[\mathrm{cm}^{-3}\right]$ is the local density of free electrons, $\kappa_{\mathrm{dust}}^{\mathrm{abs}}\left[\mathrm{cm}^{-1}\right]$ is the dust absorption coefficient, and $\chi$ is a dimensionless measure for the strength of the local UV-field

$$
\chi=\int_{91.2 \mathrm{~nm}}^{205 \mathrm{~nm}} \lambda u_{\lambda} d \lambda / \int_{91.2 \mathrm{~nm}}^{205 \mathrm{~nm}} \lambda u_{\lambda}^{\text {Draine }} d \lambda,
$$

where $\lambda u_{\lambda}=\frac{4 \pi}{c} \lambda J_{\lambda}\left[\mathrm{erg} / \mathrm{cm}^{3}\right]$ is the local photon energy density. $\lambda u_{\lambda}^{\text {Draine }}$ is an estimate of the UV radiation field in the local interstellar medium as emitted from distant $\mathrm{O}$ and B stars by Draine \& Bertoldi (1996), who have deduced these results from the original work of Draine (1978).

The efficiencies $\epsilon$ are of order 0.1 for graphite and silicates. A straightforward way to compute the PE heating rate is to compute $\chi$ from a dust continuum radiative transfer, and then to use Eq. (41), originally developed for ISM conditions. The problem in disks is that (i) the grains can be huge, a $\mathrm{mm}$-grain can easily pick up thousands of elementary charges, (ii) the UV radiation field close to the central star can be huge, for example $\chi=10^{8}$ is typically reached at a distance of about $0.1 \mathrm{AU}$ from a T Tauri star, and (iii) the wavelength range of the radiation should be extended toward the soft UV and optical, where in particular Herbig Ae/Be stars can provide vast amounts of radiation. Therefore, a full revision of the PE heating effect under disk conditions would be highly desirable, also because new laboratory results are available (e.g. Abbas et al. 2006). Surprisingly, the large grain photoelectric yields measured by Abbas et al. (2006) are much larger than theoretically expected.

Following the straightforward way to use Eq. (41) leads to quite a limited importance of the dust PE heating in protoplanetary disks. The reason is that the dust absorption coefficient per gas mass, $\kappa_{\text {dust }}^{\text {abs }} / \rho$, is lower than in the interstellar medium, by orders of magnitudes, because (we assume that) the dust grains in disks are much bigger on average.

\subsection{PAH heating}

Photoelectric effect on PolyAromatic Hydrocarbon molecules (PAHs) possibly plays a major role for the heating of the gas in protoplanetary disks. PAHs seem to have sizes of at least 100 carbon atoms in 
Summer School "Protoplanetary Disks: Theory and Modeling Meet Observations"

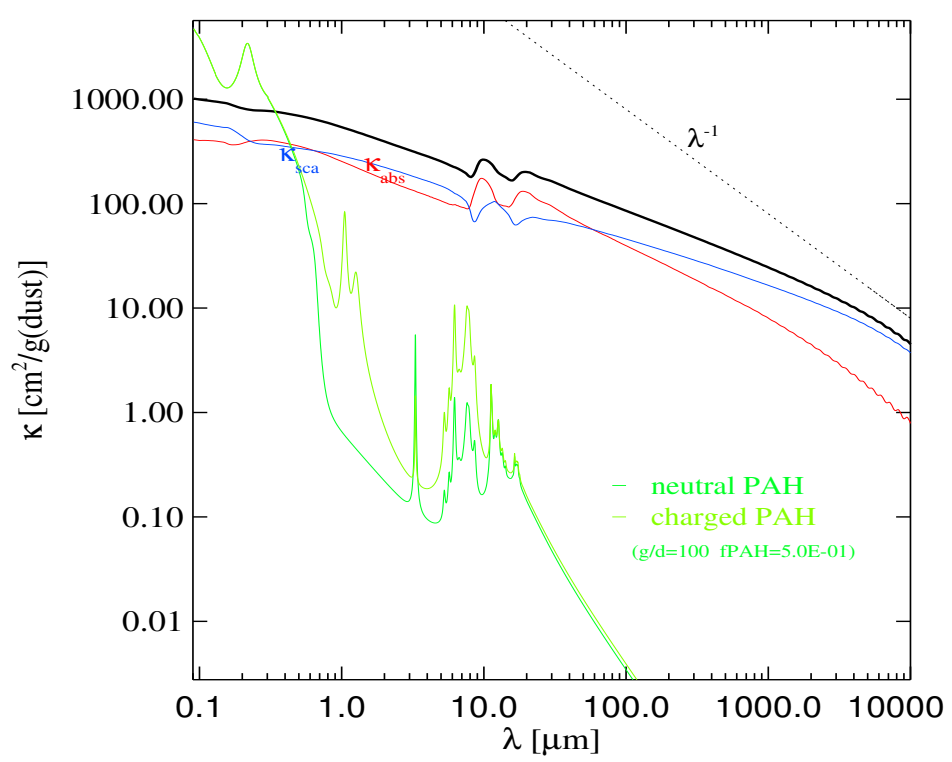

Figure 7. Comparison of dust and PAH opacities in disks. The dust opacities (black $=$ extinction, blue $=$ scattering, red = absorption) are quite low in the UV, according to our assumption of a continuous dust size distribution between $0.05 \mu \mathrm{m}$ and $3 \mathrm{~mm}$ with powerlaw index -3.5 , and dominated by the PAH opacities (green and light green represent neutral and charged PAHs). We have selected a PAH with $N_{C}=100$ carbon atoms and $N_{H}=25$ hydrogen atoms, and followed the PAH opacity model by Draine \& Li (2007). A dust/gas mass ratio of 100 is assumed, and the PAH abundance with respect to standard ISM is assumed to be $f_{\mathrm{PAH}}=0.5$.

disks (Visser et al. 2007), and to be somewhat underabundant with respect to the interstellar medium, $f_{\mathrm{PAH}} \lesssim 0.1$ (Geers et al. 2006), where $f_{\mathrm{PAH}}$ is the PAH particle abundance with respect to the standard $\mathrm{PAH}$ abundance in the ISM of Tielens (2008)

$$
n_{\mathrm{PAHs}} / n_{\langle\mathrm{H}\rangle}=3 \times 10^{-7} f_{\mathrm{PAH}} \frac{50}{N_{C}} .
$$

The physics of the PE of PAHs is very similar to the dust PE discussed in the previous section. For a selected PAH size, PAH molecules can be charged positively up to $N$ times $(N=3$ for 100 carbon atoms), can be neutral, or can be singly negatively charged. Therefore, we put species $\mathrm{PAH}^{-}$, $\mathrm{PAH}, \mathrm{PAH}^{+}, \mathrm{PAH}^{2+}, \mathrm{PAH}^{3+}(k=-1,0,1,2,3)$ as additional specimen into the chemistry, where they undergo photo-ionisation, recombination \& charge exchange reactions, with photo-ionisation rate $[1 / \mathrm{s}]$

$$
R_{\mathrm{PAH}^{k}}^{\text {photo }}=\frac{4 \pi}{c h} \int_{91.2 \mathrm{~nm}}^{\lambda_{\mathrm{thr}}^{k}} \lambda J_{\lambda} \sigma_{\mathrm{PAH}}^{k}(\lambda) Y^{k}(\lambda) d \lambda,
$$

where $\mathrm{IP}^{k}$ is the size-dependent ionisation potentials of the PAH molecules with charge $k, \lambda_{\text {thr }}$ is the threshold wavelength corresponding to $h v=\mathrm{IP}^{k}$. According to the approximate formula in Weingartner \& Draine (2001), the selected PAH with $N_{C}=100$ and $N_{H}=25$ has ionisation potentials $\mathrm{IP}^{-1}=3.32 \mathrm{eV}, \mathrm{IP}^{0}=5.85 \mathrm{eV}, \mathrm{IP}^{+1}=8.38 \mathrm{eV}$ and $\mathrm{IP}^{+2}=10.91 \mathrm{eV}$, and the triply ionised state cannot be ionised further with an FUV photon $(\lambda>91.2 \mathrm{~nm}) . \sigma_{\mathrm{PAH}}^{k}\left[\mathrm{~cm}^{2}\right]$ is the PAH absorption cross section as 

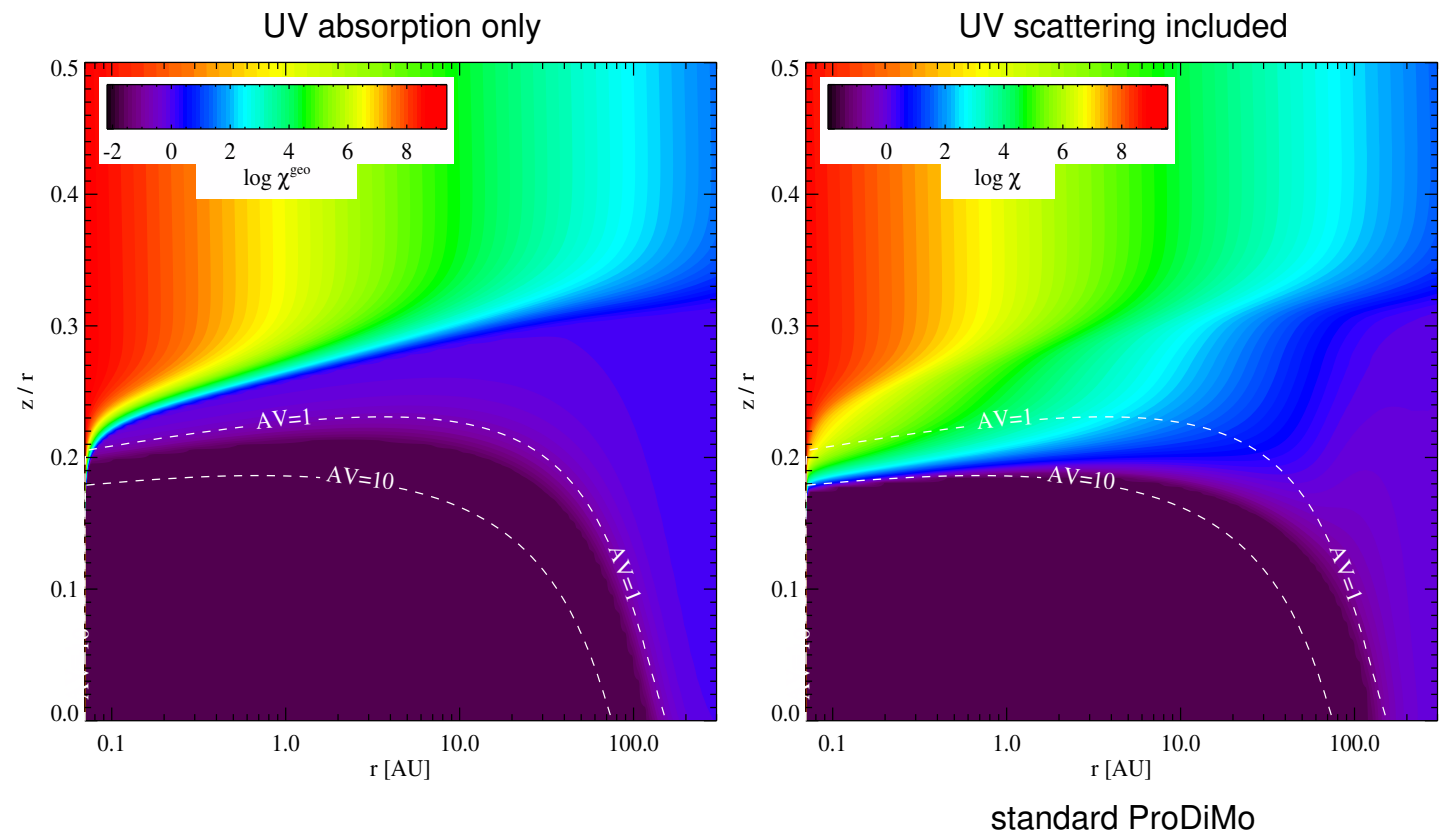

Figure 8. The UV radiation field strength relative to ISM standard conditions, $\chi$, as defined in Eq. (42), as function of position $(r, z)$ in a typical T Tauri disk model. The 1.h.s. shows the results of a simplified UV radiative transfer model, where only dust absorption is taken into account, the r.h.s. shows the solution of a full dust radiative transfer including dust scattering. The white dashed contour lines show the vertical $A_{V}$.

shown in Fig. 7, and $Y^{k}$ is the PE yield (see e.g. Tielens 2008). The PAH photoelectric heating rate and recombination cooling rate are then given by

$$
\begin{aligned}
\Gamma_{\mathrm{PAH}} & =\frac{4 \pi}{h c} \sum_{k} n_{\mathrm{PAH}}^{k} \int_{91.2 \mathrm{~nm}}^{\lambda_{\mathrm{thr}}^{k}} \lambda J_{\lambda} \sigma_{\mathrm{PAH}}^{k}(\lambda) Y^{k}(\lambda)\left(h v-\mathrm{IP}^{k}\right) d \lambda \\
\Lambda_{\mathrm{PAH}} & =\sum_{k} n_{\mathrm{PAH}}^{k} n_{e} R_{\mathrm{rec}}^{k}\left(T_{\mathrm{g}}\right) \frac{3}{2} k T_{\mathrm{g}},
\end{aligned}
$$

where the $\left(1.5 \mathrm{k} T_{\mathrm{g}}\right)$ is an approximation for $\left\langle E_{\mathrm{th}}\right\rangle$, the mean thermal energy disappearing from the gas phase due to a recombination process, see Sect. 3.

\subsection{UV and X-ray radiative transfer}

All high-energy heating processes discussed in this section (Sect. 6) are driven by UV and X-ray photons as present at the location of interest. In other words, we must know the local UV radiation field ( $J_{v}$ between $91.2 \mathrm{~nm}$ and about $400 \mathrm{~nm}$, or simply $\chi$, see Eq. 42) and the local primary X-ray ionisation rate $\zeta_{\text {Xray }}$, respectively. These quantities are affected by absorption and scattering in the disk, so the question arises how, and how deep, these high energy photons penetrate the disk. Since disks are usually optically thick in the UV as well as in X-rays, we require the solution of a full radiative transfer in the FUV and X-ray spectral regions, including scattering. 


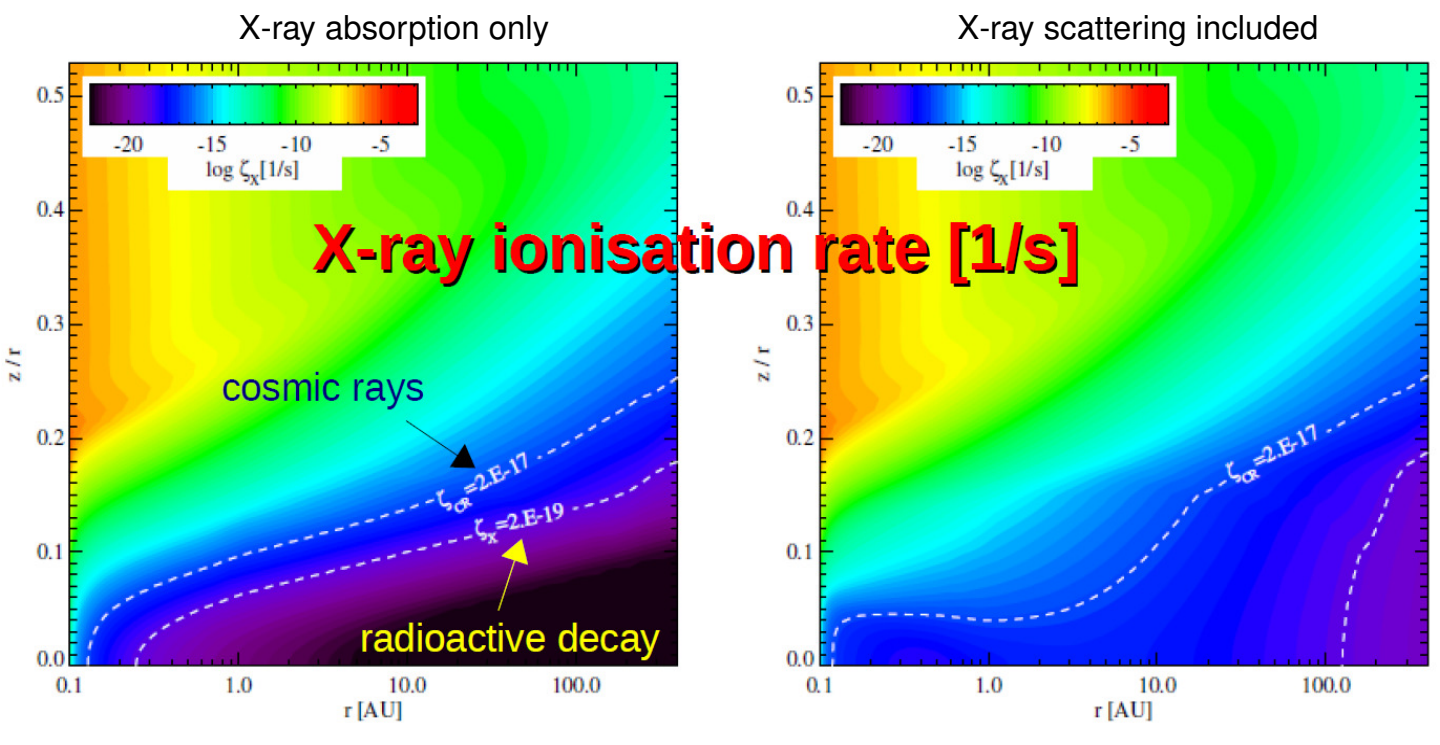

standard ProDiMo

Christian Rab, PhD thesis, Vienna University

Figure 9. The primary X-ray ionisation rate $\zeta$ as function of position $(r, z)$ in a typical T Tauri disk model. The 1.h.s. diagram shows the results if only gas absorption is taken into account, the r.h.s. shows results from a full $\mathrm{X}$-ray radiative transfer which includes gas (e.g. Compton) scattering. The white dashed lines show where the ionisation by cosmic rays and by radioactive decay, respectively, become more important than by X-rays. The $\mathrm{X}$-ray source is a point source at $(0,0)$ in the model.

The UV radiative transfer is mostly dominated by the dust, which can be easily included in the continuum radiative transfer ${ }^{2}$. Figure 8 demonstrates the importance of UV dust scattering. In a pure dust absorption model (1.h.s.), the local UV intensities would be very low indeed, the line forming regions (typically between about $A_{V}=0.01$ and $A_{V}=1$ ) would be very cold. However, UV photons can reach the disk indirectly by scattering, the mainstream pathway of a UV photon reaching the disk involves at least one of two scattering events! A typical UV photon hitting the disk is a UV photon that has been emitted by the star, was scattered by small dust particles in the optically thin disk surface layer, and approaches the disk from the top. Consequently, as shown on the r.h.s. of Fig. 8, the local UV strength $\chi$ is much larger than expected in the line forming regions, the difference can easily be as large as 6 orders of magnitude.

Bottom line is, the UV radiation field in disks is vastly different from interstellar clouds. The UV radiation source (the central T Tauri star) is very close and strong, and has a different characteristic colour, and lines, as compared to the standard ISM UV field. Due to the scattered nature of UV and Xray photons reaching the disk, the disk geometry plays a crucial role for the temperature determination in the line forming regions. This raises some uncomfortable questions when trying to take over heating formulae from interstellar cloud and PDR research into disk models.

One more factor of uncertainty, maybe the largest factor altogether, is the role of UV gas opacities in disks. Since dust particles in disks are expected to be much bigger on average, due to grain coagulation in the disk, the UV dust opacities are reduced by several orders of magnitude with respect to the

\footnotetext{
${ }^{2}$ However, standard Monte-Carlo techniques usually don't pay much attention to these wavelength, because they are not meaningful for the dust energy budget, we have modified both MCFOST and MCMax to compute the UV radiation field with higher precision, i.e. less MC noise.
} 
interstellar medium (see Fig. 7). Hence, PAHs or molecules may provide the dominant source of UV opacities. Usually, in PDR research, the influence of such gas opacities is considered to be "small", and the corrections with respect to a pure dust radiative transfer model are handled by (self-) shielding factors according to the gas column densities towards the UV source. But clearly, if those opacities become dominant, this concept brakes down, and the continuum transfer should contain these opacities consistently. This is the topic of ongoing research, very much work in progress.

The problem is challenging, because the UV gas opacities depend on chemical concentrations, hence chemistry and radiative transfer must be solved in an iterative, consistent way. Bethell \& Bergin (2011), argue, for example, that Ly-alpha resonance scattering in the tenuous disc surface layers leads to an amplification of photo-dissociation in discs, because the Ly-alpha photons emitted from the star can be re-directed in a large volume around the disk, with selective destruction of $\mathrm{HCN}, \mathrm{NH}_{3}$ and $\mathrm{CH}_{4}$, which have non-zero photo-coss-sections at $121.6 \mathrm{~nm}$, and an enhancement of $\mathrm{CO}, \mathrm{CN}$, and SO.

The X-ray radiative transfer is driven by the gas absorption and scattering opacities of either the abundant $\mathrm{H}$ and $\mathrm{He}$ atoms, and by the heavy ions like Fe, depending on X-ray energy. Scattering turns out to be just as important for X-rays as for UV photons (see Fig. 9). The key process is Compton scattering, in particular the high-energy X-rays are scattered quite efficiently. One might think that Compton scattering is highly in-elastic and angle-dependent, however this is true for $\gamma$-rays only. Even at $E=10 \mathrm{keV}$, which is quite a "hard" wavelength for X-rays, the scattering phase function is not so much different from the usual gas Rayleigh scattering phase function. Models with X-ray radiative transfer must involve iterations, because the abundance of the opacity carriers is a result of the chemistry, and the opacities determine the local X-ray intensities.

\section{Heating processes involving $\mathrm{H}_{2}$}

A number of follow-up processes occur if the abundant $\mathrm{H}_{2}$ molecules absorb a FUV photon, see black arrow in Fig. 10, resulting in a $\mathrm{H}_{2}$ molecule in an electronically excited state.

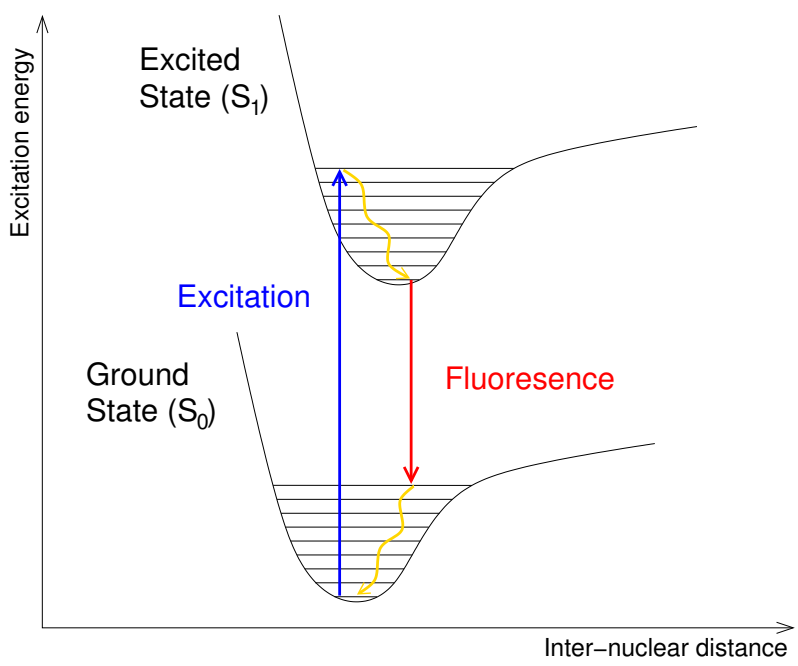

Figure 10. Processes occurring after $\mathrm{H}_{2}$ is excited by a FUV photon into an electronically excited state. Vertical axis is excitation energy, horizontal axis is inter-nuclear distance. 


\section{1 $\mathrm{H}_{2}$ photo-dissociation heating}

The photodissociation of $\mathrm{H}_{2}$ is triggered by the absorption of FUV radiation in the Werner \& Lyman UV line series, exciting the molecule to an electronic excited, but still bound state. Fast radiative (infrared) vibrational de-excitation leads to an increases of the inter-nuclear distance (Fig. 10). Finally, the electronically excited state will emit another UV photon which brings the $\mathrm{H}_{2}$ back to it's electronic ground state. This state is highly excited (vibrationally), and in about $10 \%$ of all cases, the molecules ends up in an unbound state which dissociates. The two liberated $\mathrm{H}$-atoms are known to carry away about $0.4 \mathrm{eV}$ in form of kinetic energy (the mean energy excess of the $S_{0}$ states as fluorescently populated from above over the $E(R \rightarrow \infty)$-value in Fig. 10, see Stephens \& Dalgarno 1973). The heating rate due to $\mathrm{H}_{2}$ photodissociation is hence

$$
\Gamma_{\mathrm{ph}}^{\mathrm{H}_{2}}=6.4 \times 10^{-13} R_{\mathrm{ph}}^{\mathrm{H}_{2}} n_{\mathrm{H}_{2}}
$$

where $R_{\mathrm{ph}}^{\mathrm{H}_{2}}$ is the $\mathrm{H}_{2}$ photo-dissociation rate $\propto \chi$, including $\mathrm{H}_{2}$ self-shielding factors.

\subsection{Excited $\mathrm{H}_{2}$ de-excitation}

In $90 \%$ of all cases, however, after the absorption on an FUV photon, the electronically excited $\mathrm{H}_{2}$ molecule returns to a bound, though highly vibrationally excited state in the electronic ground level. This state will either further decay radiatively (in which case there is no further impact on the thermal energy of the gas), or collisionally. In the latter case, the vibrational excitation energy is thermalised, and we have a heating rate as

$$
\begin{aligned}
\Gamma_{\text {coll }}^{\mathrm{H}_{2}^{\star}} & =\Delta E R_{\mathrm{H}_{2}^{\star} \rightarrow \mathrm{H}_{2}}^{\text {coll }}\left(n_{\mathrm{H}_{2}^{\star}}-n_{\mathrm{H}_{2}} \exp \left(-\frac{\Delta E}{k T_{\mathrm{g}}}\right)\right) \\
R_{\mathrm{H}_{2}^{\star} \rightarrow \mathrm{H}_{2}}^{\text {coll }} & =n_{\mathrm{H}} C_{u l}^{\mathrm{H}}\left(T_{\mathrm{g}}\right)+n_{\mathrm{H}_{2}} C_{u l}^{\mathrm{H}_{2}}\left(T_{\mathrm{g}}\right) .
\end{aligned}
$$

The formulation of the heating rate by collisional excitation of vibrationally excited $\mathrm{H}_{2}$ follows Tielens \& Hollenbach (1985) where one pseudo vibration level with energy difference $\Delta E \approx 2.6 \mathrm{eV}$ is introduced, which is fluorescently pumped by FUV radiation and has a radiative lifetime of about $\sim 280 \mathrm{yrs}\left(\mathrm{H}_{2}\right.$ quadrupole lines!). The collisional de-excitation rates $C_{u l}$, for collisions with $\mathrm{H}$ and $\mathrm{H}_{2}$, are also given in Tielens \& Hollenbach (1985). The second term in Eq. (48) corrects for collisional excitation (cooling). In order to compute the particle densities of the excited and ground state molecule, $n_{\mathrm{H}_{2}}^{\star}$ and $n_{\mathrm{H}_{2}}$, respectively, $\mathrm{H}_{2}^{\star}$ is included in the chemistry, where the rate of fluorescent formation of $\mathrm{H}_{2}^{\star}$ is given by $10 \times$ the photo-dissociation rate.

\section{3 $\mathrm{H}_{2}$ formation heating}

When molecular hydrogen forms on dust surfaces, the $\mathrm{H}_{2}$ binding energy of $4.48 \mathrm{eV}$ is released. This energy will partly heat the dust grain, and will partly be distributed over rotational, vibrational and kinetic energy of the escaping $\mathrm{H}_{2}$ molecule. The latter fraction means heating of the gas. Duley \& Williams (1986) carried out a theoretical study of the details of the formation process, and concluded that the escaping $\mathrm{H}_{2}$ molecule should be kinetically and vibrationally excited, but rotationally cool. For simplicity, if we assume that about $1 / 3$ of the energy goes into translation, i.e. about $1.5 \mathrm{eV}$ (Black \& Dalgarno 1976), the $\mathrm{H}_{2}$ formation heating rate is given by

$$
\Gamma_{\text {form }}^{\mathrm{H}_{2}}=2.39 \times 10^{-12} R_{\mathrm{H}_{2}} n_{\mathrm{H}}
$$




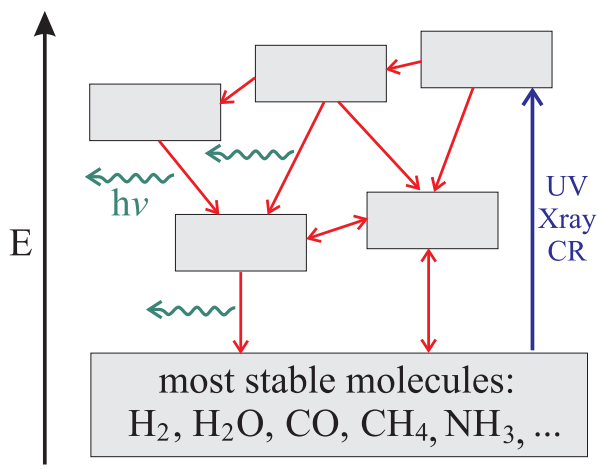

Figure 11. Schematic energetics in disk chemistry (from Woitke et al. 2011, reproduced with permission (c) ESO). The most abundant stable molecules are attacked by high- $E$ radiation, leading to energetically less stable species. As these fragments recombine and react chemically to re-form the stable molecules, binding energy is liberated which may be radiated away (green wiggled arrows) or may heat the gas (red arrows).

where $R_{\mathrm{H}_{2}}[1 / s]$ is the $\mathrm{H}_{2}$ formation rate on grain surfaces and $n_{\mathrm{H}}$ is the particle density of atomic hydrogen.

Just as the $\mathrm{H}_{2}$ photo-dissociation heating and the $\mathrm{H}_{2}^{\star}$ collisional de-excitation heating, the $\mathrm{H}_{2}$ formation heating can be considered as a follow-up process of UV photons hitting $\mathrm{H}_{2}$, because that's the key process to destroy the $\mathrm{H}_{2}$ molecule in the first place, before it can again recombine on dust surfaces. In other words, without UV photons, there is only very little $\mathrm{n}_{\mathrm{H}}$.

\section{Chemical heating and cooling}

The previous section provided two examples for how various follow-up collisional, radiative and chemical processes, after the initial absorption of a UV photon by an $\mathrm{H}_{2}$ molecule, can lead to heating while finally re-installing chemical equilibrium. These two examples (the heating by collisional deexcitation of $\mathrm{H}_{2}^{\star}$, and the heating by $\mathrm{H}_{2}$ formation on grains) show interesting ways how to partly thermalise incoming UV photon energy.

This reveals a more general principle as sketched in Fig. 11. In kinetic chemical equilibrium, there is actually no net formation/destruction of any chemical species, but the chemistry is organised in never ending, complicated reaction cycles. These cycles are powered by incoming UV-photons, $\mathrm{X}$-ray photons or cosmic rays. If all high- $E$ sources would be taken away, and if the gas is cold, the chemistry would eventually become very pure, with only the most stable molecules with closed shells being abundant, such as $\mathrm{H}_{2}, \mathrm{CO}, \mathrm{CH}_{4}, \mathrm{~N}_{2}$, and $\mathrm{NH}_{3}$, and practically no reactions occurring at all.

However, since the stable molecules are continuously attacked by high-energy radiation, some of those molecules ionise or form smaller reactive fragments which are, in sum, energetically less favourable. Subsequent chemical reactions will then stepwise re-form the stable molecules along complicated paths. The overwhelming majority of those reactions are "exothermic", i.e. they release heat. Some parts of that surplus energy will be lost in form of secondary photons, but the main effect is clear. We formulate the "chemical heating" rate as

$$
\Gamma_{\text {chem }}=\sum_{r} R(r) \gamma_{r}^{\text {chem }} \Delta H_{r}
$$


where $r$ is the index of a gas-phase chemical reaction, $R(r)$ is the corresponding reaction rate $\left[1 / \mathrm{cm}^{3} / \mathrm{s}\right]$, and

$$
\Delta H_{r}=\sum_{\mathrm{pr}} \Delta H_{f}^{0}(\mathrm{pr})-\sum_{\mathrm{ed}} \Delta H_{f}^{0}(\mathrm{ed}) .
$$

is the net reaction enthalpy, i.e. the net heat production per reaction. $\Delta H_{f}(\mathrm{ed})$ and $\Delta H_{f}(\mathrm{pr})$ are the formation enthalpies of the involved educts and products. To simplify, we take the formation enthalpies at zero temperature from Millar et al. (1997) instead. Is it important to evaluate the sum in Eq. (51) only for pure gas phase reaction, and stay away from any reactions that have cosmic rays, dust, or photons on either side of the reaction, also from reactions whose heating rates are treated explicitly elsewhere, to avoid double accounting. $\gamma_{r}^{\text {chem }}$ is a thermalisation efficiency of the reaction, which is usually unknown, but the examples in the last section suggests that $1 / 3$ might be a typical value, even higher if the energy going into rotational and vibrational excitation of the products is thermalised by collisions afterwards.

If the gas is hot, several $1000 \mathrm{~K}$, endothermic reactions consume energy to surmount the high activation barriers involved into these reactions. These reactions, for example "collider" reactions, cool the gas with high efficiency $\gamma_{r}^{\text {chem }} \approx 1$. This is automatically taken into account in Eq. (51) which states the net heating rate $=$ (heating - cooling) rate.

The chemical heating is yet another way to partially thermalise the energy of an incoming UV photon, X-ray photon or a cosmic ray particle, through subsequent exothermic chemical reactions. These reaction may take place a long time after the primary interaction took place. The chemical heating, even with low efficiency $\gamma^{\text {chem }}=0.1$, results to be an important heating process in protoplanetary disks, in particular at the bottom of the warm molecular layer where many of the near-mid IR spectral lines are formed, and densities are of order $10^{9}-10^{13} \mathrm{~cm}^{-3}$.

\section{Viscous Heating}

Mass accretion onto the star implies a slow inward motion of the gas with velocity $v(r, z)$ as

$$
\dot{M}(r)=2 \pi r \int_{-\infty}^{+\infty} \rho(r, z) v(r, z) d z .
$$

Let us study the energetic consequence of that slow inward motion, assuming that the gas stays on close-to Keplerian orbits. For simplicity, we assume here that the mass accretion rate $\dot{M}(r)=$ const throughout the disk, and we assume that the velocity does not depend on height $z$, i.e. $v(r, z)=v(r)$, in which case Eq. (53) simplifies as

$$
\dot{M}=2 \pi r \Sigma(r) v(r) .
$$

The gravitational potential energy per surface area of a small annulus $\left[\mathrm{erg} / \mathrm{cm}^{2}\right]$ positioned at radius $r$, in the thin disk approximation, is given by

$$
E_{\mathrm{pot}}(t)=-\frac{G M_{\star} \Sigma(r)}{r}
$$

During a small time interval $d t$, the position $r$ of that annulus shrinks as $r \rightarrow r-v(r) d t$, hence the potential energy changes as

$$
E_{\mathrm{pot}}(t+d t)=-\frac{G M_{\star} \Sigma(r)}{r-v(r) d t} \approx-\frac{G M_{\star} \Sigma(r)}{r^{2}}(r+v(r) d t)=E_{\mathrm{pot}}(t)-\frac{G M_{\star}}{r^{2}} \Sigma(r) v(r) d t
$$


Now, using $\Sigma(r) v(r)=\dot{M} /(2 \pi r)($ Eq. 54), we find

$$
\frac{d E_{\mathrm{pot}}}{d t}=-\frac{G M_{\star} \dot{M}}{2 \pi r^{3}}
$$

On Keplerian orbits, the kinetic energy per surface area is

$$
E_{\mathrm{kin}}(t)=\frac{1}{2} \Sigma(r) v_{\mathrm{Kep}}^{2}(r)=\frac{1}{2} \Sigma(r) \frac{G M_{\star}}{r}
$$

Assuming the gas to always quickly re-adjust to Keplerian velocities, we find

$$
E_{\mathrm{kin}}(t+d t)=\frac{1}{2} \Sigma(r) \frac{G M_{\star}}{r-v(r) d t} \approx \frac{1}{2} \Sigma(r) \frac{G M_{\star}}{r^{2}}(r+v(r) d t)=E_{\mathrm{kin}}(t)+\frac{1}{2} \frac{G M_{\star}}{r^{2}} \Sigma(r) v(r) d t
$$

Hence,

$$
\frac{d E_{\mathrm{kin}}}{d t}=+\frac{G M_{\star} \dot{M}}{4 \pi r^{3}} .
$$

Summing up the changes of potential and kinetic energies (Eqs. 57 and 60), it becomes clear that $E_{\text {pot }}+E_{\text {kin }}$ must decrease if the gas is to move inwards on close-to Keplerian orbits. Where does that liberated energy go? If the disk is isolated from the environment, we can assume $E_{\text {pot }}+E_{\text {kin }}+E_{\text {therm }}=$ const, hence the net decrease of $E_{\text {pot }}+E_{\text {kin }}$ must be compensated for by an increase of the internal energy of the gas in the annulus $E_{\text {therm. }}$. An alternative way to understand this phenomenon is to realise that the establishment of a new Keplerian orbit at a smaller radius $r$ requires the action of a braking force. In disk isolation, we need an internal force to do that job. How exactly this works in nature is not clear, but the classical idea is to assume that a frictional force is at action, and to describe it like a viscous force (Shakura \& Syunyaev 1973). Therefore, the increase of $E_{\text {therm }}$ is called the viscous heating rate

$$
\frac{d}{d t} E_{\mathrm{therm}}=-\frac{d E_{\mathrm{pot}}}{d t}-\frac{d E_{\mathrm{kin}}}{d t}=\frac{G M_{\star} \dot{M}}{4 \pi r^{3}} .
$$

A more thorough derivation, using viscous evolution theory, is given in Armitage (2007). The difference is that $\dot{M}$ is actually not constant, and calibrated at the stellar surface (see Armitage 2007, their Eqs. 65 and 71). Their result is

$$
\frac{d}{d t} E_{\text {therm }}=\frac{3 G M_{\star} \dot{M}}{8 \pi r^{3}}\left(1-\sqrt{\frac{R_{\star}}{r}}\right)
$$

which differs by a factor of 2/3 from our simple derivation, times a meaningless factor if $r \gg R_{\star}$. Equation (62) forms the basis of the d'Alessio disk models (D'Alessio et al. 1998). Note that in Eq. (62) there is actually no dimensionless "alpha" viscosity $\alpha_{\text {vis }}$. The $1 / r^{3}$-factor in Eq. (62) is important. It means that the inner disks of classical T Tauri stars, which have much smaller inner radii, are much more affected by viscous heating as compared to Herbig Ae/Be stars.

Now, for $2 \mathrm{D}$ models, we need to turn this heating rate per column $\left[\mathrm{erg} / \mathrm{cm}^{2} / \mathrm{s}\right]$ into a heating rate per volume $\left[\mathrm{erg} / \mathrm{cm}^{3} / \mathrm{s}\right.$ ], i.e. we need to make an assumption about how that total heating rate is distributed within the column as function of height $z$. Let's assume we choose

$$
\Gamma_{\mathrm{vis}}(r, z)=\frac{3 G M_{\star} \dot{M}}{8 \pi r^{3}}\left(1-\sqrt{\frac{R_{\star}}{r}}\right) \frac{\rho^{p}(r, z)}{\int \rho^{p}\left(r, z^{\prime}\right) d z^{\prime}} .
$$

where, obviously, $\int \Gamma_{\text {vis }}(r, z) d z=\frac{d}{d t} E_{\text {therm. }}$. From linear theory of viscosity, $p=1$ would be suggested (see e.g. Frank et al. 1992), but turbulent energy dissipation is not necessarily linear. Assuming $p=1$ 

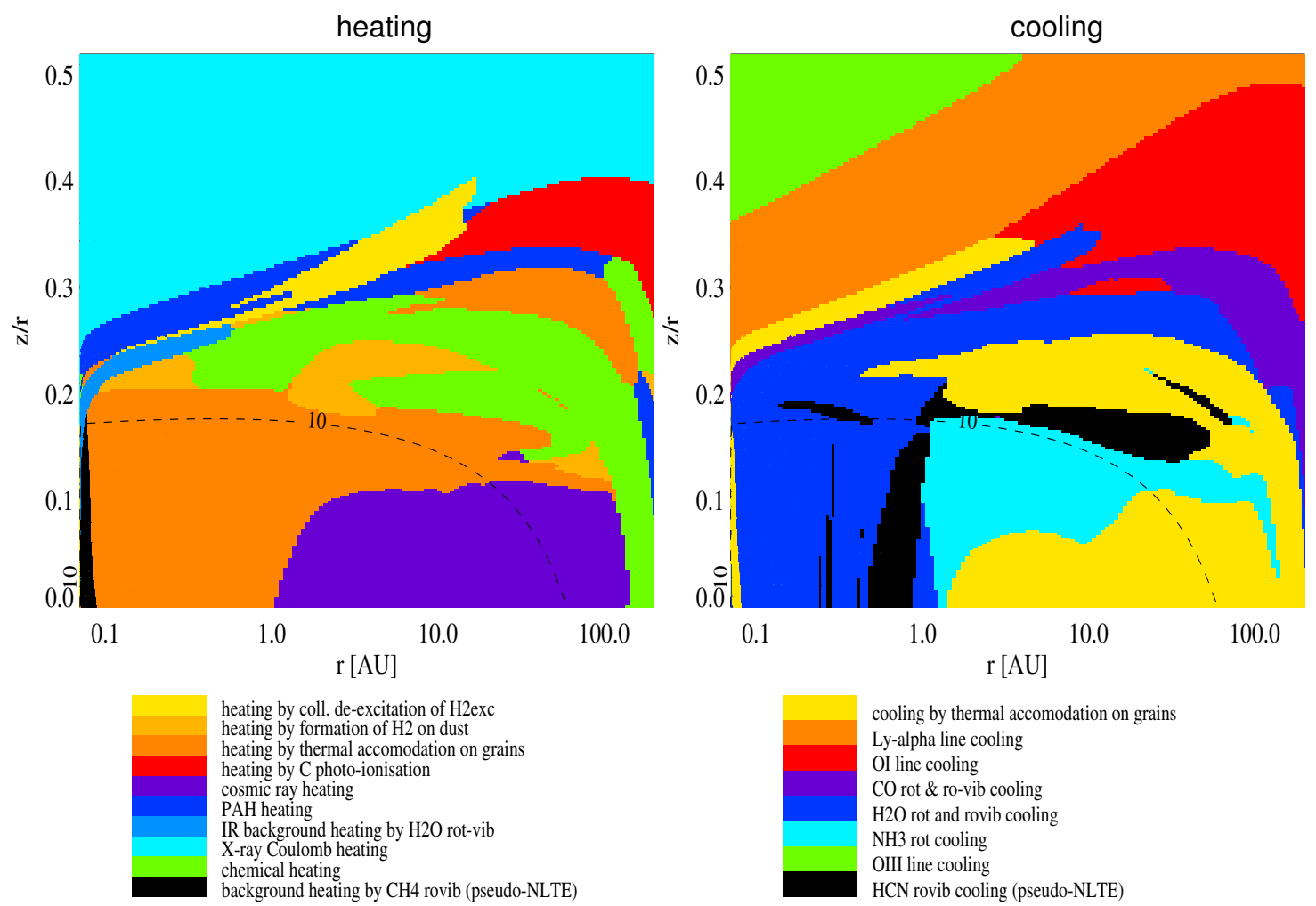

Figure 12. Leading heating and cooling processes as function of position $(r, z)$ in the disk. The dashed black line marks optical extinction $A_{V}=10$.

leads to unstoppable heating for $\rho \rightarrow 0$ high above the disk midplane, because we then have a heating rate $\Gamma \propto \rho$ whereas all cooling processes approach the non-LTE limit $\Lambda \propto \rho^{2}$. Instead, $p=2$ does work, but contradicts the linear theory of viscosity.

A solution of this puzzle requires to understand and evaluate the viscosity behind the viscous heating. If we assign the origin of the viscosity to the magneto-rotational instability (MRI), a detailed treatment of non-ideal MHD effects (resistivity, ambipolar diffusion ...) based on the computed ionisation degree in disks (Thi et al. 2015, in prep.) shows that the ambipolar diffusion reduces the heating effect in very thin plasmas, in which case the above mentioned inconsistency does not occur.

\section{Results}

We conclude this chapter by looking at some results obtained by the ProDiMo code to a standard $\mathrm{T}$ Tauri disk model setup. The setup is designed such that the model predicts continuum and line fluxes that roughly resemble the observations of real class II T Tauri stars (shape of SED, mm-flux and slope, [OI] $63 \mu \mathrm{m}$ line flux, etc.). The effective stellar temperature is chosen as $T_{\text {eff }}=4000 \mathrm{~K}$, and the stellar luminosity $L_{\star}=1 L_{\odot}$. These values correspond to spectral type K7, a stellar mass of $M_{\star}=0.7 M_{\odot}$ and an age of about 1.6 Myrs. For more details about this model setup, please study http://www.diana-project.com/data-results-downloads/an-example-disc-model/. 
EPJ Web of Conferences
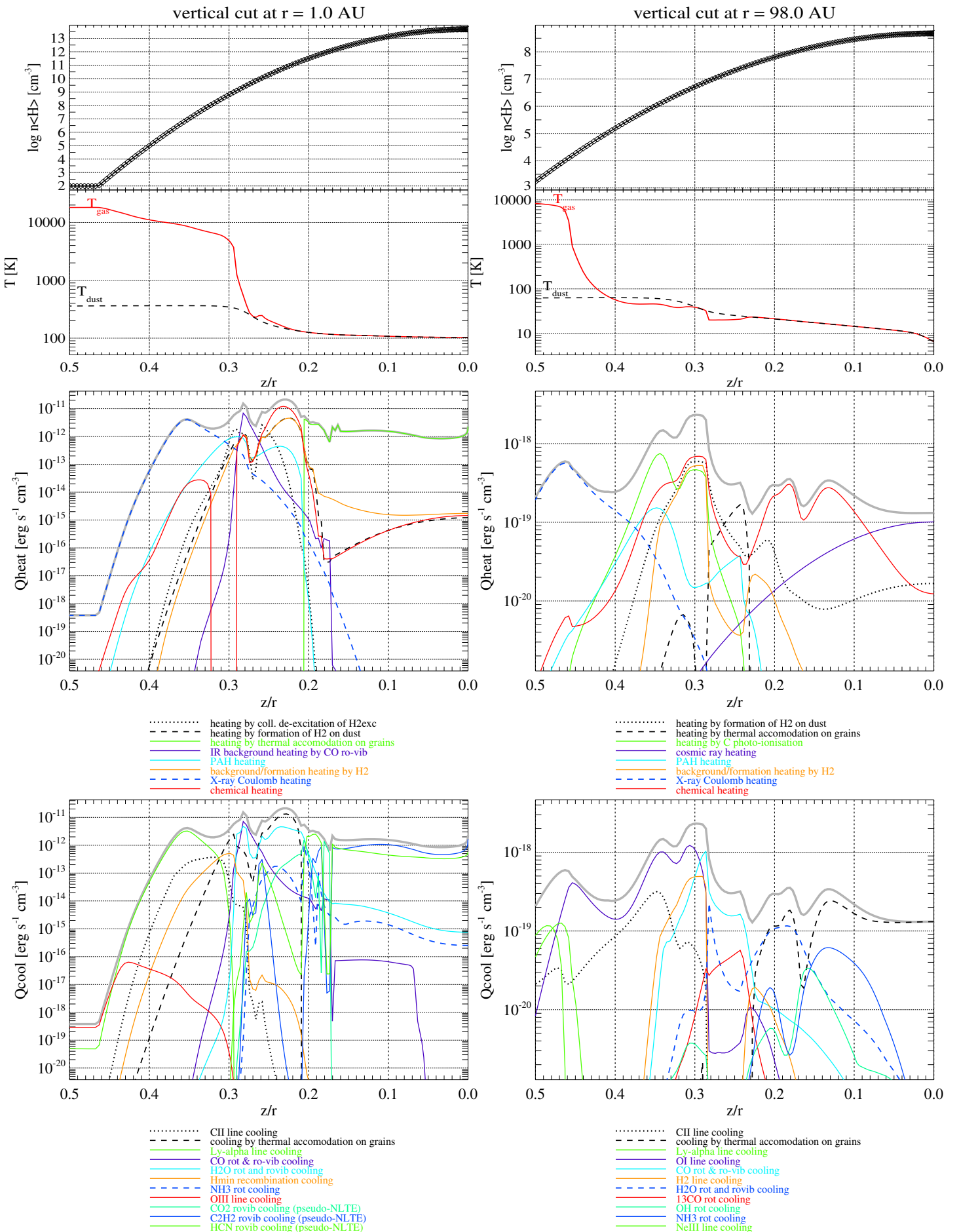

Figure 13. Vertical cuts at $r=1 \mathrm{AU}$ (1.h.s.) and $r=100 \mathrm{AU}$ (r.h.s.). The upper figures show the assumed density profiles, and the calculated dust and gas temperature structures. The second and third rows show the most important heating and cooling rates. Note that, at every point, the total heating rate equals the total cooling rate. 


\section{Summer School "Protoplanetary Disks: Theory and Modeling Meet Observations"}

Figure 12 gives an overview of the leading heating and cooling processes as function of radial and vertical position in the disk. Figure 13 shows some more details in form of vertical cuts at $1 \mathrm{AU}$ and $100 \mathrm{AU}$. We can roughly distinguish between three different vertical layers.

(i) The hot atomic layer: The top layer of the disk surface results to be extremely hot ( $T_{\text {gas }}>$ $10000 \mathrm{~K}$ ), an ionised plasma which is heated by X-rays, and cools via O III and Ly $\alpha$ line emission. However, this region is so tenuous, that is doesn't produce any observable line signatures, maybe except for some [OI] $6300 \AA$ and [NeII] $12.82 \mu \mathrm{m}$ emission.

(ii) The warm molecular layer: The vertical temperature and chemical disk structure, at any radius, resembles an (X-ray) photo-dissociation region (PDR, X-PDR), see Fig. 13. As the incoming UV and $\mathrm{X}$-ray photons start to be absorbed (by dust and gas above, and by molecular self-shielding), first molecules like $\mathrm{H}_{2}$ and $\mathrm{CO}$ can form, with $\mathrm{H}_{2} \mathrm{O}$ forming in slightly deeper layers. These molecules introduce new and very strong line cooling functions which lead to an almost sudden decrease of the gas temperature to a few $100 \mathrm{~K}$, or a few $10 \mathrm{~K}$, depending on radius. The cooling amplifies and stabilises the molecule formation. The transition from the hot atomic to the warm molecular layer can be very sudden, with gas temperatures being either a few $1000 \mathrm{~K}$ or a few $100 \mathrm{~K}$ (a few $10 \mathrm{~K}$ ), but nothing in-between. In the warm molecular layer, UV driven heating processes, such as PAH heating, chemical heating, neutral carbon photo-ionisation, and the heating processes involving $\mathrm{H}_{2}$ are balanced by line emission. The warm molecular layer is characterised by a very active photo and X-ray chemistry (fast chemical relaxation) and elevated gas temperatures $T_{\mathrm{g}}>T_{\mathrm{d}}$. It is mainly responsible for the observable line emissions, in particular concerning the near-IR and mid-IR.

Figure 13 (see cooling plots at the bottom) allows for a direct estimate of where most of the line emissions are generated, and which species are most important. At $1 \mathrm{AU}$, a first peak of $Q_{\text {cool }}$ occurs at $z / r=0.28\left(n_{\langle\mathrm{H}\rangle} \approx 10^{9} \mathrm{~cm}^{-3}\right)$ due to ro-vibrational cooling of $\mathrm{CO}$ and $\mathrm{H}_{2} \mathrm{O}$, just where these molecules start to form. We receive ro-vibrational emission from the very top of the warm molecular layer. A second maximum exists around $z / r=0.23\left(n_{\langle\mathrm{H}\rangle} \approx 10^{11} \mathrm{~cm}^{-3}\right)$ mainly due to rotational cooling by $\mathrm{H}_{2} \mathrm{O}$.

At $100 \mathrm{AU},[\mathrm{OI}] 63 \mu \mathrm{m}$ cooling dominates above the warm molecular layer, and rotational cooling of $\mathrm{CO}$ and $\mathrm{H}_{2} \mathrm{O}$ are most important in the warm molecular layer. Since the cooling lines are typically all optically thick, the emission region can be confined to a quite thin spatial zone characterised by large temperature gradients, a numerical challenge. In fact, there is quite a distinctive maximum at $z / r=0.3\left(n_{\langle\mathrm{H}\rangle} \approx 10^{7} \mathrm{~cm}^{-3}\right)$.

(iii) The icy midplane: The third and deepest layer is reached when the optical extinction reaches about $A_{V}=10$. No UV photons, and not much X-rays reach this region, and the gas temperature equilibrates with the dust temperature through thermal accommodation, leading to $T_{\mathrm{g}} \approx T_{\mathrm{d}}$. At large enough distances, where the midplane dust temperatures are sufficiently low, molecules freeze out (forming in particular ices of $\mathrm{H}_{2} \mathrm{O}, \mathrm{CO}, \mathrm{CO}_{2}, \mathrm{CH}_{4}$ and $\mathrm{NH}_{3}$ ), which soon consume all available oxygen, carbon, and nitrogen from the gas phase, leaving $\mathrm{H}_{2}$ as the only abundant molecule in the distant midplane (Helling et al. 2014). This limiting case, however, is only reached for midplane dust temperature $\lesssim 20 \mathrm{~K}$, but also not too distant from the star where the disk becomes transparent to UV radiation again, which prevents ice formation due to UV photo-desorption.

A further remarkable feature at smaller radii (see 1.h.s. of Fig. 13) is that, at such large densities, the heating/cooling behaviour of the gas becomes LTE-like just as in stellar atmospheres. There are layers where $\mathrm{CO}$ ro-vib is both the most important heating and the most important cooling process, so the gas is approximately in radiative equilibrium, as explained in Sect. 1.3, with respect to the CO line opacities. Closer inspection shows that rather a combination of $\mathrm{CO}, \mathrm{H}_{2} \mathrm{O}, \mathrm{HCN}, \mathrm{CO}_{2}, \mathrm{NH}_{3}, \mathrm{CH}_{4}$ and $\mathrm{C}_{2} \mathrm{H}_{2}$ line opacities is what counts, just like in brown dwarf atmospheres (see e.g. Helling \& Casewell 2014; Helling \& Woitke 2006). 
Another interesting result is shown on the r.h.s. of Fig. 13. In the distant layers situated deep enough, which are barely reached by any UV or X-rays, the line cooling (e.g. CO) is so effective that it actually leads to $T_{\text {gas }}<T_{\text {dust }}$. Here, the gas is heated by thermal accommodation, and cools via line emission.

Acknowledgements I want to thank Wing-Fai Thi for his scientific input and critical reading of the manuscript. The research leading to these results has received funding from the European Union Seventh Framework Programme FP7-2011 under grant agreement no 284405.

\section{References}

Abbas, M. M., Tankosic, D., Craven, P. D., et al. 2006, ApJ, 645, 324

Armitage, P. J. 2007, ArXiv Astrophysics e-prints

Avrett, E. H. \& Hummer, D. G. 1965, MNRAS, 130, 295

Bakes, E. L. O. \& Tielens, A. G. G. M. 1994, ApJ, 427, 822

Bethell, T. J. \& Bergin, E. A. 2011, ApJ, 739, 78

Black, J. H. \& Dalgarno, A. 1976, ApJ, 203, 132

Bruderer, S., van der Marel, N., van Dishoeck, E. F., \& van Kempen, T. A. 2014, A\&A, 562, A26

Burke, J. R. \& Hollenbach, D. J. 1983, ApJ, 265, 223

Cleeves, L. I., Adams, F. C., \& Bergin, E. A. 2013, ApJ, 772, 5

Cleeves, L. I., Bergin, E. A., Qi, C., Adams, F. C., \& Öberg, K. I. 2015, ApJ, 799, 204

D’Alessio, P., Canto, J., Calvet, N., \& Lizano, S. 1998, ApJ, 500, 411

Dalgarno, A., Yan, M., \& Liu, W. 1999, ApJSS, 125, 237

Draine, B. T. 1978, ApJSS, 36, 595

Draine, B. T. \& Bertoldi, F. 1996, ApJ, 468, 269

Draine, B. T. \& Li, A. 2007, ApJ, 657, 810

Duley, W. W. \& Williams, D. A. 1986, MNRAS, 223, 177

Frank, J., King, A., \& Raine, D. 1992, Accretion Power in Astrophysics (Accretion Power in Astrophysics, ISBN 0521408636, Cambridge University Press, 1992.)

Fujii, Y. I., Okuzumi, S., \& Inutsuka, S. 2011, ApJ, 743, 53

Geers, V. C., Augereau, J.-C., Pontoppidan, K. M., et al. 2006, A\&A, 459, 545

Glassgold, A. E., Galli, D., \& Padovani, M. 2012, ApJ, 756, 157

Gorti, U. \& Hollenbach, D. 2009, ApJ, 690, 1539

Groenewegen, M. A. T. 1994, A\&A, 290, 531 
Summer School "Protoplanetary Disks: Theory and Modeling Meet Observations"

Güdel, M. 2015, in EPJ Web of Conferences, Vol. 102, Summer School on Protoplanetary Disks: Theory and Modeling Meet Observations, ed. I. Kamp, P. Woitke, \& J. D. Ilee

Helling, C. \& Casewell, S. 2014, A\&ARv, 22, 80

Helling, C. \& Woitke, P. 2006, A\&A, 455, 325

Helling, C., Woitke, P., Rimmer, P. B., et al. 2014, Life, 4, 142

Hogerheijde, M. R. \& van der Tak, F. F. S. 2000, A\&A, 362, 697

Hollenbach, D. \& McKee, C. F. 1979, ApJSS, 41, 555

Hollenbach, D. \& McKee, C. F. 1989, ApJ, 342, 306

Hummer, D. G. 1988, ApJ, 327, 477

Ilee, J. D., Boley, A. C., Caselli, P., et al. 2011, MNRAS, 417, 2950

John, T. L. 1994, MNRAS, 269, 871

Kamp, I. 2015, in EPJ Web of Conferences, Vol. 102, Summer School on Protoplanetary Disks: Theory and Modeling Meet Observations, ed. I. Kamp, P. Woitke, \& J. D. Ilee

Kamp, I., Tilling, I., Woitke, P., Thi, W., \& Hogerheijde, M. 2010, A\&A, 510, A260000+

McElroy, D., Walsh, C., Markwick, A. J., et al. 2013, A\&A, 550, A36

Mihalas, D. 1978, Stellar atmospheres /2nd edition/ (San Francisco, W. H. Freeman and Co.)

Millar, T. J., Farquhar, P. R. A., \& Willacy, K. 1997, A\&AS, 121, 139

Min, M. 2015, in EPJ Web of Conferences, Vol. 102, Summer School on Protoplanetary Disks: Theory and Modeling Meet Observations, ed. I. Kamp, P. Woitke, \& J. D. Ilee

Rybicki, G. B. \& Lightman, A. P. 1979, Radiative processes in astrophysics (New York, WileyInterscience, 1979. 393 p.)

Shakura, N. I. \& Syunyaev, R. A. 1973, A\&A, 24, 337

Shu, F. H. 1992, Physics of Astrophysics, Vol. II (University Science Books)

Somerville, W. B. 1964, ApJ, 139, 192

Stephens, T. L. \& Dalgarno, A. 1973, ApJ, 186, 165

Stilley, J. L. \& Callaway, J. 1970, ApJ, 160, 245

Tielens, A. G. G. M. 2008, ARA\&A, 46, 289

Tielens, A. G. G. M. \& Hollenbach, D. 1985, ApJ, 291, 747

Umebayashi, T. \& Nakano, T. 2009, ApJ, 690, 69

Visser, R., Geers, V. C., Dullemond, C. P., et al. 2007, A\&A, 466, 229

Weingartner, J. C. \& Draine, B. T. 2001, ApJSS, 134, 263 


\section{EPJ Web of Conferences}

Woitke, P. 1997, PhD thesis, Technische Univervität Berlin

Woitke, P., Kamp, I., \& Thi, W.-F. 2009, A\&A, 501, 383

Woitke, P., Krueger, D., \& Sedlmayr, E. 1996, A\&A, 311, 927

Woitke, P., Riaz, B., Duchêne, G., et al. 2011, A\&A, 534, A44 